Review Article

\title{
A Review on Conduction Mechanisms in Dielectric Films
}

\author{
Fu-Chien Chiu \\ Department of Electronic Engineering, Ming Chuan University, Taoyuan 333, Taiwan \\ Correspondence should be addressed to Fu-Chien Chiu; fcchiu@mail.mcu.edu.tw
}

Received 29 August 2013; Revised 5 December 2013; Accepted 11 December 2013; Published 18 February 2014

Academic Editor: Chun-Hsing Shih

Copyright (C) 2014 Fu-Chien Chiu. This is an open access article distributed under the Creative Commons Attribution License, which permits unrestricted use, distribution, and reproduction in any medium, provided the original work is properly cited.

\begin{abstract}
The conduction mechanisms in dielectric films are crucial to the successful applications of dielectric materials. There are two types of conduction mechanisms in dielectric films, that is, electrode-limited conduction mechanism and bulk-limited conduction mechanism. The electrode-limited conduction mechanism depends on the electrical properties at the electrode-dielectric interface. Based on this type of conduction mechanism, the physical properties of the barrier height at the electrode-dielectric interface and the effective mass of the conduction carriers in dielectric films can be extracted. The bulk-limited conduction mechanism depends on the electrical properties of the dielectric itself. According to the analyses of bulk-limited conduction mechanisms, several important physical parameters in the dielectric films can be obtained, including the trap level, the trap spacing, the trap density, the carrier drift mobility, the dielectric relaxation time, and the density of states in the conduction band. In this paper, the analytical methods of conduction mechanisms in dielectric films are discussed in detail.
\end{abstract}

\section{Introduction}

The application of dielectric films has always been a very important subject for the semiconductor industry and the scientific community. This is especially true for metal-oxidesemiconductor field effect transistor (MOSFET) technology in integrated circuits (ICs). The concept of MOSFET is based on the modulation of channel carriers by an applied gate voltage across a thin dielectric. Dielectric is a material in which the electrons are very tightly bonded. The electric charges in dielectrics will respond to an applied electric field through the change of dielectric polarization. Dielectric materials are nearly insulators in which the electrical conductivity is very low and the energy band gap is large. In general, the value of energy band gap of insulators is set to be larger than $3 \mathrm{eV}$ or $5 \mathrm{eV}$. Although not all dielectrics are insulators, all insulators are typical dielectrics. At $0 \mathrm{~K}$, the valence band is completely filled and the conduction band is completely empty. Thus, there is no carrier for electrical conduction. When the temperature is larger than $0 \mathrm{~K}$, there will be some electrons thermally excited from the valence band and also from the donor impurity level to the conduction band. These electrons will contribute to the current transport of the dielectric material. Similarly, holes will be generated by acceptor impurities and vacancies will be left by excited electrons in the valence band. The conduction current of insulators at normal applied electric field will be very small because their conductivities are inherently low, on the order of $10^{-20} \sim 10^{-8} \Omega^{-1} \mathrm{~cm}^{-1}$. However, the conduction current through the dielectric film is noticeable when a relatively large electric field is applied. These noticeable conduction currents are owing to many different conduction mechanisms, which is critical to the applications of the dielectric films. For example, the gate dielectric of MOSFETs, the capacitor dielectric of dynamic random access memories, and the tunneling dielectric of Flash memories are of top importance to the IC applications. In these cases, the conduction current must be lower than a certain level to meet the specific reliability criteria under normal operation of the devices. Consequently, the study of the various conduction mechanisms through dielectric films is of great importance to the success of the integrated circuits.

To measure the conduction current through the dielectric film, one must prepare some kind of sample devices for testing. In general, there are two types of device structures used in sample testing. One is metal-insulator-metal structure which is called the MIM capacitor or the MIM diode. The considered issue in MIM capacitors is the possible asymmetry of the electrical properties when the top and bottom electrodes 


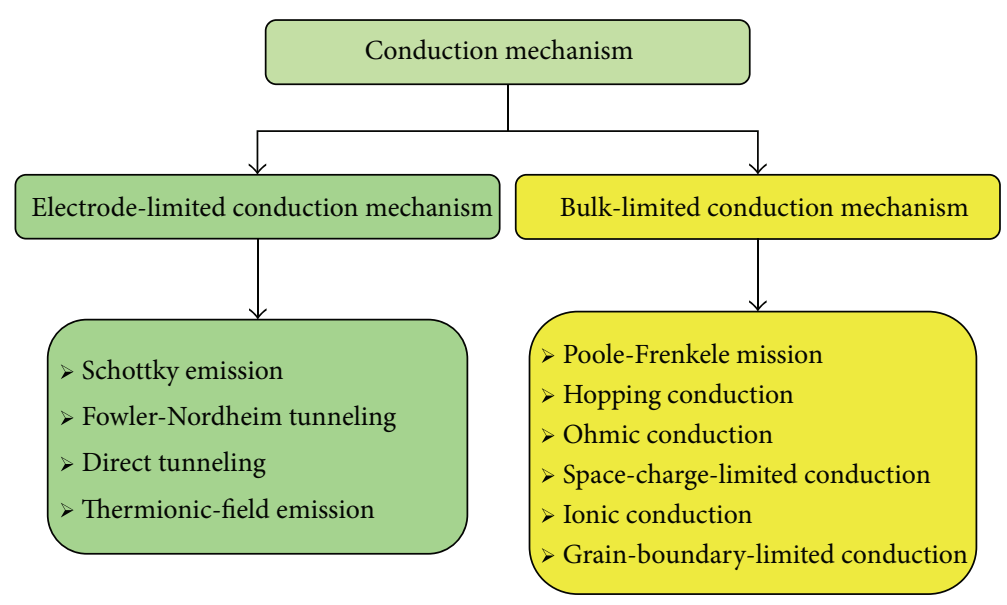

FIGURE 1: Classification of conduction mechanisms in dielectric films.

are made of different metals. Different metals generally lead to different work functions, and therefore result in different metal-dielectric interface barriers. The main parameters in this type of measurement are the barrier height of the metaldielectric interface and the effective mass of the conduction carriers. The second type used to characterize a dielectric film is the metal-insulator-semiconductor (MIS) capacitor. Since MIS capacitor is the most useful device in the study of semiconductor surfaces, it is of interest to characterize the electrical properties of the device. However, the structure of the MIS capacitor is inherently asymmetric and one should be careful about the voltage drop across each layer. If the MIS capacitor can be biased in such a way that the semiconductor surface is in accumulation, the voltage drop across the semiconductor is minimal and most of the voltage will be applied across the dielectric film. If the semiconductor surface is in depletion or inversion, some voltage drop across the semiconductor will take place and then the voltage drop needs to be considered in calculation of the electric field across the dielectric film.

Among the conduction mechanisms being investigated, some depend on the electrical properties at the electrodedielectric contact. These conduction mechanisms are called electrode-limited conduction mechanisms or injection-limited conduction mechanisms. There are other conduction mechanisms which depend only on the properties of the dielectric itself. These conduction mechanisms are called bulk-limited conduction mechanisms or transport-limited conduction mechanisms [1-10]. The methods to distinguish these conduction mechanisms are essential because there are a number of conduction mechanisms that may all contribute to the conduction current through the dielectric film at the same time. Since several conduction mechanisms depend on the temperature in different ways, measuring the temperature dependent conduction currents may afford us a helpful way to know the constitution of the conduction currents. The electrode-limited conduction mechanisms include (1) Schottky or thermionic emission (2) Fowler-Nordheim tunneling, (3) direct tunneling, and (4) thermionic-field emission. The bulk-limited conduction mechanisms include (1) Poole-Frenkel emission, (2) hopping conduction, (3) ohmic conduction, (4) space-charge-limited conduction, (5) ionic conduction, and (6) grain-boundary-limited conduction. Figure 1 shows the classification of conduction mechanisms in dielectric films.

\section{Electrode-Limited Conduction Mechanisms}

The electrode-limited conduction mechanisms depend on the electrical properties at the electrode-dielectric contact. The most important parameter in this type of conduction mechanism is the barrier height at the electrodedielectric interface. The electrode-limited conduction mechanisms include (1) Schottky or thermionic emission, (2) Fowler-Nordheim tunneling, (3) direct tunneling, and (4) thermionic-field emission. The current due to thermionic emission is highly dependent on the temperature, whereas the tunneling current is nearly temperature independent. Aside from the barrier height at the electrode-dielectric interface, the effective mass of the conduction carriers in dielectric films is also a key factor in the electrode-limited conduction mechanisms.

2.1. Schottky or Thermionic Emission. Schottky emission is a conduction mechanism that if the electrons can obtain enough energy provided by thermal activation, the electrons in the metal will overcome the energy barrier at the metaldielectric interface to go to the dielectric. Figure 2 shows the MIS energy band diagram when the metal electrode is under negative bias with respect to the dielectric and the semiconductor substrate. The energy barrier height at the metaldielectric interface may be lowered by the image force. The barrier-lowering effect due to the image force is called Schottky effect. Such a conduction mechanism due to electron emission from the metal to the dielectric is called thermionic emission or Schottky emission. Thermionic emission is one of 


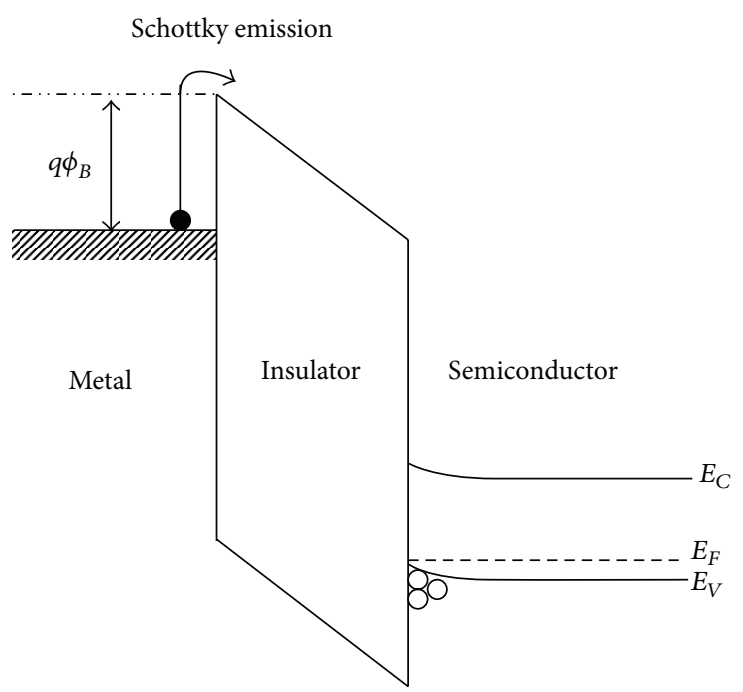

Figure 2: Schematic energy band diagram of Schottky emission in metal-insulator-semiconductor structure.

the most often observed conduction mechanism in dielectric films, especially at relatively high temperature. The expression of Schottky emission is

$$
\begin{array}{r}
J=A^{*} T^{2} \exp \left[\frac{-q\left(\phi_{B}-\sqrt{q E / 4 \pi \varepsilon_{r} \varepsilon_{0}}\right)}{k T}\right], \\
A^{*}=\frac{4 \pi q k^{2} m^{*}}{h^{3}}=\frac{120 m^{*}}{m_{0}},
\end{array}
$$

where $J$ is the current density, $A^{*}$ is the effective Richardson constant, $m_{0}$ is the free electron mass, $m^{*}$ is the effective electron mass in dielectric, $T$ is the absolute temperature, $q$ is the electronic charge, $q \phi_{B}$ is the Schottky barrier height (i.e., conduction band offset), $E$ is the electric field across the dielectric, $k$ is the Boltzmann's constant, $h$ is the Planck's constant, $\varepsilon_{0}$ is the permittivity in vacuum, and $\varepsilon_{r}$ is the optical dielectric constant (i.e., the dynamic dielectric constant). In view of the classical relation between dielectric and optical coefficients, the dynamic dielectric constant should be close to the square of the optical refractive index (i.e., $\varepsilon_{r}=$ $n^{2}$ ) [11]. It is worthy of note that the dielectric constant is generally a function of frequency, why the optical dielectric constant is used in this case. During the emission process, if the electron transit time from the metal-dielectric interface to the barrier maximum position is shorter than the dielectric relaxation time, the dielectric does not have enough time to be polarized; consequently, the dielectric constant at high frequency or optical dielectric constant should be chosen. This optical dielectric constant is smaller than the static dielectric constant or the value at low frequency where more polarization mechanisms can contribute to the total polarization [10].

Figure 3 shows the current density-electric field $(J-E)$ characteristics of $\mathrm{Al} / \mathrm{CeO}_{2} / p$-Si MIS capacitors biased in

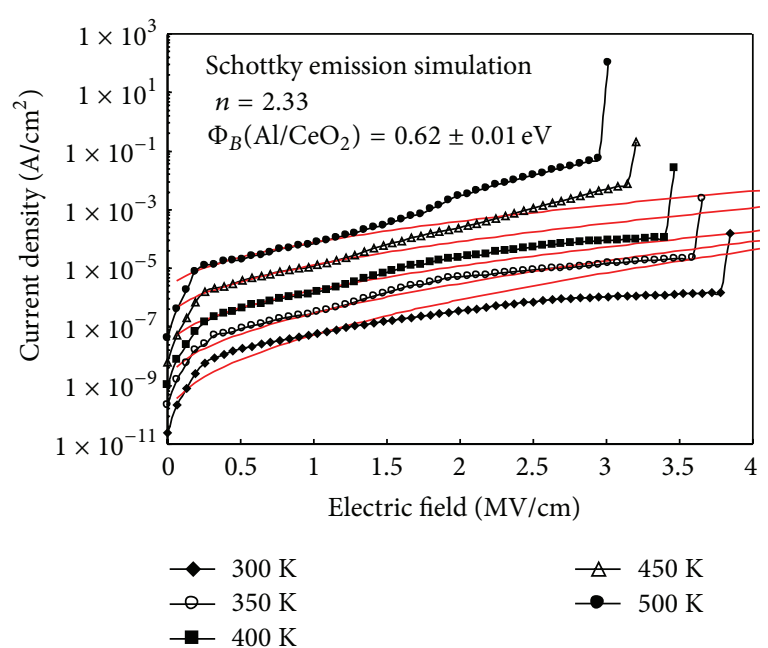

FIGURE 3: Experimental $J-E$ curves (symbols) and simulation of Schottky emission (lines) for the $\mathrm{Al} / \mathrm{CeO}_{2} / p$-Si MIS capacitor.

accumulation mode at temperatures ranging from $300 \mathrm{~K}$ to $500 \mathrm{~K}$. According to the optical characterization of $\mathrm{CeO}_{2}$ films, the refractive index $(n)$ at $632.8 \mathrm{~nm}$ is about 2.33 . Therefore, the optical dielectric constant of $\mathrm{CeO}_{2}$ films is about 5.43. The measured $J-E$ curves in [11] and the simulations of Schottky emission are shown in Figure 3. The experimental data match the Schottky emission theory very well at high temperature $(\geq 400 \mathrm{~K})$ in a medium electric field $(0.5 \sim 1.6 \mathrm{MV} / \mathrm{cm})$. The corresponding conduction band offset between $\mathrm{Al}$ and $\mathrm{CeO}_{2}$ is then determined to be $0.62 \pm 0.01 \mathrm{eV}$.

Besides the simulation method, Schottky plot is the most popular way to identify the barrier height at the interface. For a standard Schottky emission, the plot of $\log \left(J / T^{2}\right)$ versus $E^{1 / 2}$ should be linear. The barrier height can be obtained from the intercept of Schottky plot. For example, the $J-E$ data measured at high temperatures $(>425 \mathrm{~K})$ and in high fields $(>1 \mathrm{MV} / \mathrm{cm})$ correlate well with the Schottky emission theory under the gate injection in an $\mathrm{Al} / \mathrm{ZrO}_{2}(17.4 \mathrm{~nm}) / p$-Si MIS capacitor [13], as shown in Figure 4. Moreover, the fitted optical dielectric constant in the plot of standard Schottky emission is extremely close to the square of optical refractive index (i.e., $6.25=2.5^{2}$ ), and the extracted Schottky barrier height between $\mathrm{Al}$ and $\mathrm{ZrO}_{2}$ is about $0.92 \mathrm{eV}$.

Simmons indicated that if the electronic mean free path in the insulator is less than the thickness of dielectric film, the equation of standard Schottky emission must be modified [14]. When excited electrons pass through dielectric films, the thermal electrons are affected by traps and interface states, which are generated from oxygen vacancies and thermal instability between dielectric and $\mathrm{Si}$, respectively. The thickness of dielectric films also affects the behavior of Schottky emission. A trap-limited mechanism governs the carrier transportation in dielectric films [13]. Therefore, 


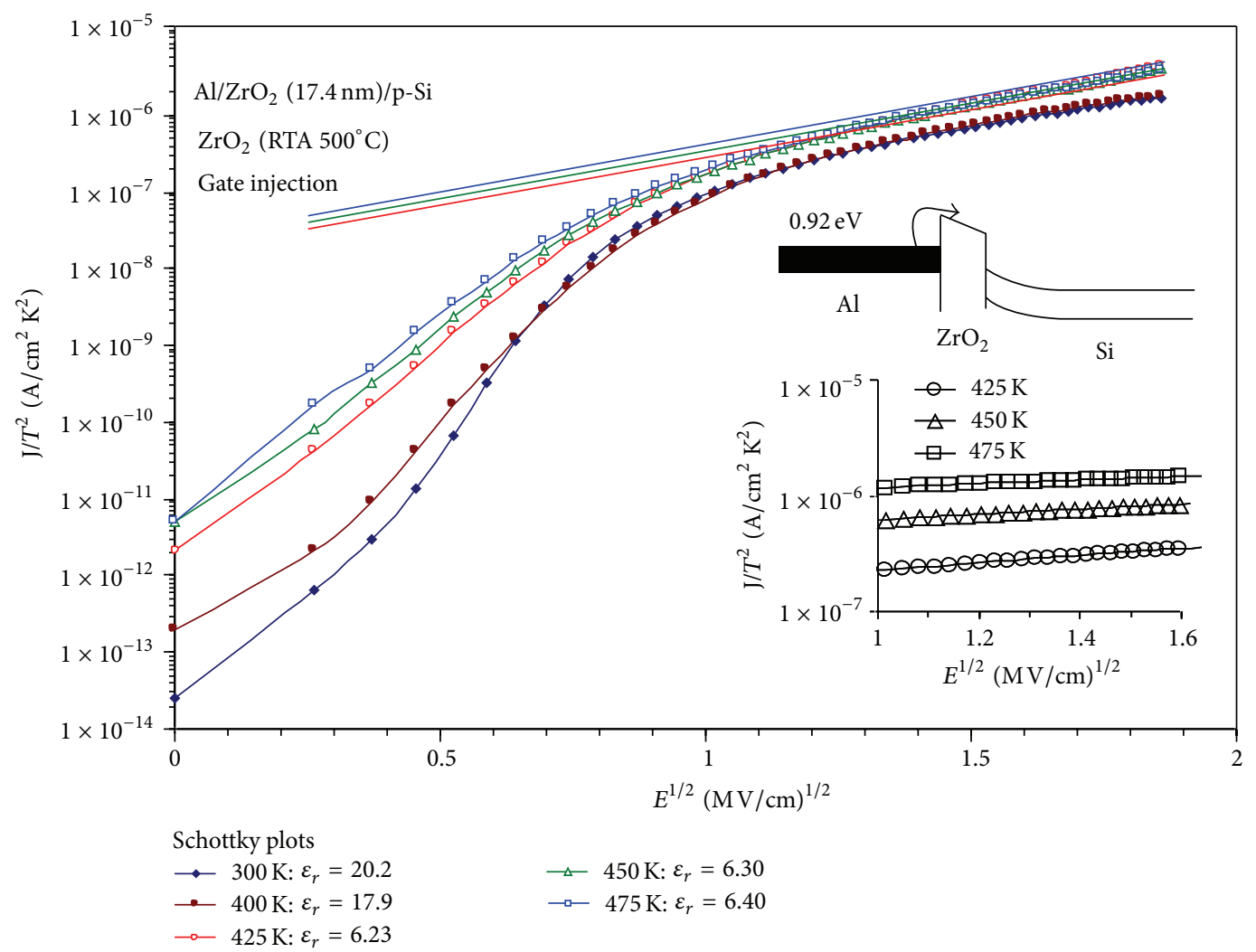

FIGURE 4: Characteristics of standard Schottky emission at various temperatures. Inset graphs present standard Schottky emission constrained by $n=\varepsilon_{r}^{1 / 2}$ at high temperatures; the band diagram is also shown.

(1) should be modified into (2) when the electronic mean free path $(l)$ is less than the dielectric thickness $\left(t_{d}\right)$ :

$$
\begin{array}{r}
J=\alpha T^{3 / 2} E \mu\left(\frac{m^{*}}{m_{0}}\right)^{3 / 2} \exp \left[\frac{-q\left(\phi_{b}-\sqrt{q E / 4 \pi \varepsilon_{r} \varepsilon_{0}}\right)}{K T}\right] \\
\left(l<t_{d}\right),
\end{array}
$$

where $\alpha=3 \times 10^{-4} \mathrm{~A} \mathrm{~s} / \mathrm{cm}^{3} \mathrm{~K}^{3 / 2}$ and $\mu$ is the electronic mobility in the insulator; the other notations are the same as defined before. Notably, no clear distinction can be made between the bulk- and electrode-limited conduction mechanisms, as indicated by (2), because each is involved in the conduction process [15]. By generating modified Schottky emission plots for $\mathrm{ZrO}_{2}$ films of various thicknesses, the electronic mean free path in $\mathrm{ZrO}_{2}$ films can be determined to be between 16.2 and $17.4 \mathrm{~nm}$ at high temperature $(>425 \mathrm{~K})$ [13]. In addition, the electronic mobility $(\mu)$ in $\mathrm{ZrO}_{2}$ films can be determined from the intercept of the plot of modified Schottky emission. The obtained electronic mobility in $\mathrm{ZrO}_{2}$ is $12-13 \mathrm{~cm}^{2} / \mathrm{V}$-s in a medium field at high temperatures [13].

2.2. Fowler-Nordheim Tunneling. According to the classical physics, when the energy of the incident electrons is less than the potential barrier, the electrons will be reflected. However, quantum mechanism predicts that the electron wave function will penetrate through the potential barrier when the barrier is thin enough $(<100 \AA)$. Hence, the probability of electrons existing at the other side of the potential barrier is not zero because of the tunneling effect. Figure 5 shows the schematic energy band diagram of Fowler-Nordheim (F-N) tunneling. $\mathrm{F}-\mathrm{N}$ tunneling occurs when the applied electric field is large enough so that the electron wave function may penetrate through the triangular potential barrier into the conduction band of the dielectric. The expression of the F-N tunneling current is

$$
J=\frac{q^{3} E^{2}}{8 \pi h q \phi_{B}} \exp \left[\frac{-8 \pi\left(2 q m_{T}^{*}\right)^{1 / 2}}{3 h E} \phi_{B}^{3 / 2}\right],
$$

where $m_{T}^{*}$ is the tunneling effective mass in dielectric; the other notations are the same as defined before. To extract the tunneling current, one can measure the current-voltage $(I-V)$ characteristics of the devices at very low temperature. At such a low temperature, the thermionic emission is suppressed and the tunneling current is dominant.

For F-N tunneling, a plot of $\ln \left(J / E^{2}\right)$ versus $1 / E$ should be linear. Figure 6 shows the $I-V$ data measured at $77 \mathrm{~K}$ for an $\mathrm{HfO}_{2}$ MIS capacitor biased in the accumulation mode [16]. The inset graph indicates that the fitting of F-N tunneling 


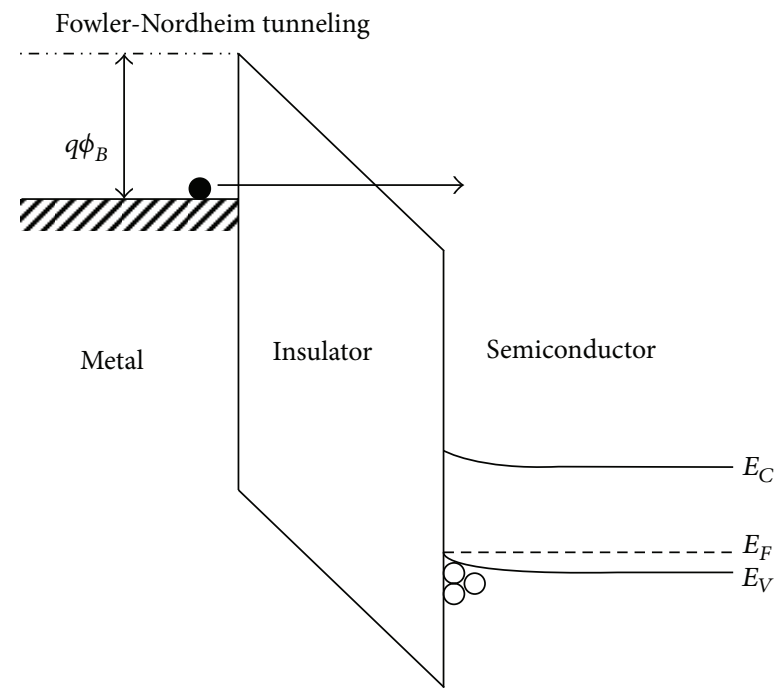

FIGURE 5: Schematic energy band diagram of Fowler-Nordheim tunneling in metal-insulator-semiconductor structure.

theory in high electric fields is very good. The slope of F$\mathrm{N}$ plot can be expressed in (4) [17] and is also a function of electron effective mass and barrier height:

$$
\text { slope }=-6.83 \times 10^{7} \sqrt{\left(\frac{m_{T}^{*}}{m_{0}}\right) \phi_{B}^{3}} .
$$

To identify electron effective mass and barrier height, it is useful to measure the thermionic emission current at high temperature and the tunneling current at low temperature. Chiu [16] reported that the electron effective mass in $\mathrm{HfO}_{2}$ and barrier height at the $\mathrm{Al} / \mathrm{HfO}_{2}$ interface can be determined using the intercept of Schottky plot at high temperatures and the slope of F-N plot at $77 \mathrm{~K}$, as shown in Figure 7. In general, $m_{T}^{*}=m^{*}$ is assumed. Using a mathematical iteration method for a $23.2 \mathrm{~nm} \mathrm{HfO} \mathrm{HIS}_{2}$ capacitor, the electron effective mass and barrier height at the $\mathrm{Al} / \mathrm{HfO}_{2}$ interface were extracted to be about $0.4 m_{0}$ and $0.94 \mathrm{eV}$, respectively [16]. These two parameters are self-consistent with the intercept of Schottky plot and the slope of F-N plot. For the case of a $12.2 \mathrm{~nm}$ $\mathrm{HfO}_{2} \mathrm{MIS}$ capacitor, the electron effective mass and $\mathrm{Al} / \mathrm{HfO}_{2}$ barrier height were determined to be $0.09 m_{0}$ and $0.94 \mathrm{eV}$, respectively.

For $\mathrm{HfO}_{2}$ films, the correlation between electron effective mass, barrier height, equivalent oxide thickness, and current conduction mechanism was summarized in [16]. In addition, reports showed that the electron effective mass in $\mathrm{SiO}_{2}$ tends to increase with decreasing oxide thickness in ultrathin silicon dioxide layers $[18,19]$. Both parabolic and nonparabolic energy-momentum dispersion were used for measuring the tunneling effective mass in $\mathrm{SiO}_{2}$. The latter has been found to work better in many cases [18-21]. As the $\mathrm{SiO}_{2}$ thickness is increased beyond $4 \mathrm{~nm}$, the nonparabolic tunneling effective mass ultimately converges to electron effective mass in $\mathrm{SiO}_{2}$ [19]. Also, the electron effective mass in $\mathrm{SiO}_{2}$ is approximately constant over the voltage bias range studied $[19,20]$. Based on these results, the tunneling effective mass can be assumed to be equal to the electron effective mass for dielectric films with wide enough thickness.

2.3. Direct Tunneling. There are two main gate current conduction mechanisms in $\mathrm{SiO}_{2}$ films. If the voltage across the $\mathrm{SiO}_{2}$ is large enough, the electrons see a triangular barrier and the gate current is due to F-N tunneling. On the other hand, if the voltage across the $\mathrm{SiO}_{2}$ is small, the electrons see the full oxide thickness and the gate current is due to directing tunneling. The driving oxide voltage between the two mechanisms is approximately $3.1 \mathrm{~V}$ for the $\mathrm{SiO}_{2}$-Si interface. For $\mathrm{SiO}_{2}$ thicknesses of 4-5 nm and above, F-N tunneling dominates and for $\mathrm{SiO}_{2}$ thickness less than about $3.5 \mathrm{~nm}$, directing tunneling becomes dominant. The schematic energy band diagram of direct tunneling is shown in Figure 8. Based on the result of Lee and $\mathrm{Hu}$ on a polysilicon- $\mathrm{SiO}_{2}$-silicon structure, the expression of the direct tunneling current density is [12]

$$
\begin{aligned}
J= & \frac{q^{2}}{8 \pi h \varepsilon \phi_{B}} C\left(V_{G}, V, t, \phi_{B}\right) \\
& \times \exp \left\{-\frac{8 \pi \sqrt{2 m^{*}}\left(q \phi_{B}\right)^{3 / 2}}{3 h q|E|} \cdot\left[1-\left(1-\frac{|V|}{\phi_{B}}\right)^{3 / 2}\right]\right\},
\end{aligned}
$$

where $t$ is the thickness of the dielectric, $V$ is the voltage across the dielectric, and the other notations are the same as defined before. The tunneling current components include electron tunneling from the conduction band (ECB), electron tunneling from the valence band (EVB), and hole tunneling form the valence band (HVB). The correction function $C$ can be expressed as [12]

$$
\begin{aligned}
& C\left(V_{G}, V, t, \phi_{B}\right) \\
& \quad=\exp \left[\frac{20}{\phi_{B}}\left(\frac{|V|-\phi_{B}}{\phi_{0}}+1\right)^{\alpha} \cdot\left(1-\frac{|V|}{\phi_{B}}\right)\right] \cdot \frac{V_{G}}{t} \cdot N,
\end{aligned}
$$

where $\alpha$ is a fitting parameter depending on the tunneling process, $q \phi_{0}$ is the $\mathrm{Si} / \mathrm{SiO}_{2}$ barrier height (e.g., $3.1 \mathrm{eV}$ for electron and $4.5 \mathrm{eV}$ for hole), and $q \phi_{B}$ is the actual barrier height (e.g., $3.1 \mathrm{eV}$ for $\mathrm{ECB}, 4.2 \mathrm{eV}$ for $\mathrm{EVB}$, and $4.5 \mathrm{eV}$ for HVB). $N$ is an auxiliary function which is used as an indicator of carrier population for ECB and HVB cases or transmission probability for EVB case. For ECB and HVB tunneling processes in both the inversion or accumulation regimes, $N$ is given by

$$
\begin{aligned}
N=\frac{\varepsilon}{t}\{ & n_{\mathrm{inv}} v_{T} \cdot \ln \left[1+\exp \left(\frac{V_{G, \mathrm{eff}}-V_{\mathrm{TH}}}{n_{\mathrm{inv}} v_{T}}\right)\right] \\
& \left.+v_{T} \cdot \ln \left[1+\exp \left(-\frac{V_{G}-V_{\mathrm{FB}}}{v_{T}}\right)\right]\right\},
\end{aligned}
$$

where $v_{T}(=k T / q)$ is the thermal voltage, $V_{\mathrm{TH}}$ is threshold voltage, $V_{\mathrm{FB}}$ is flatband voltage, and $V_{\mathrm{G} \text {,eff }}=V_{G}-V_{\text {poly }}$ is the effective gate voltage after accounting for the voltage drop across the polysilicon depletion region. The rate of increase 


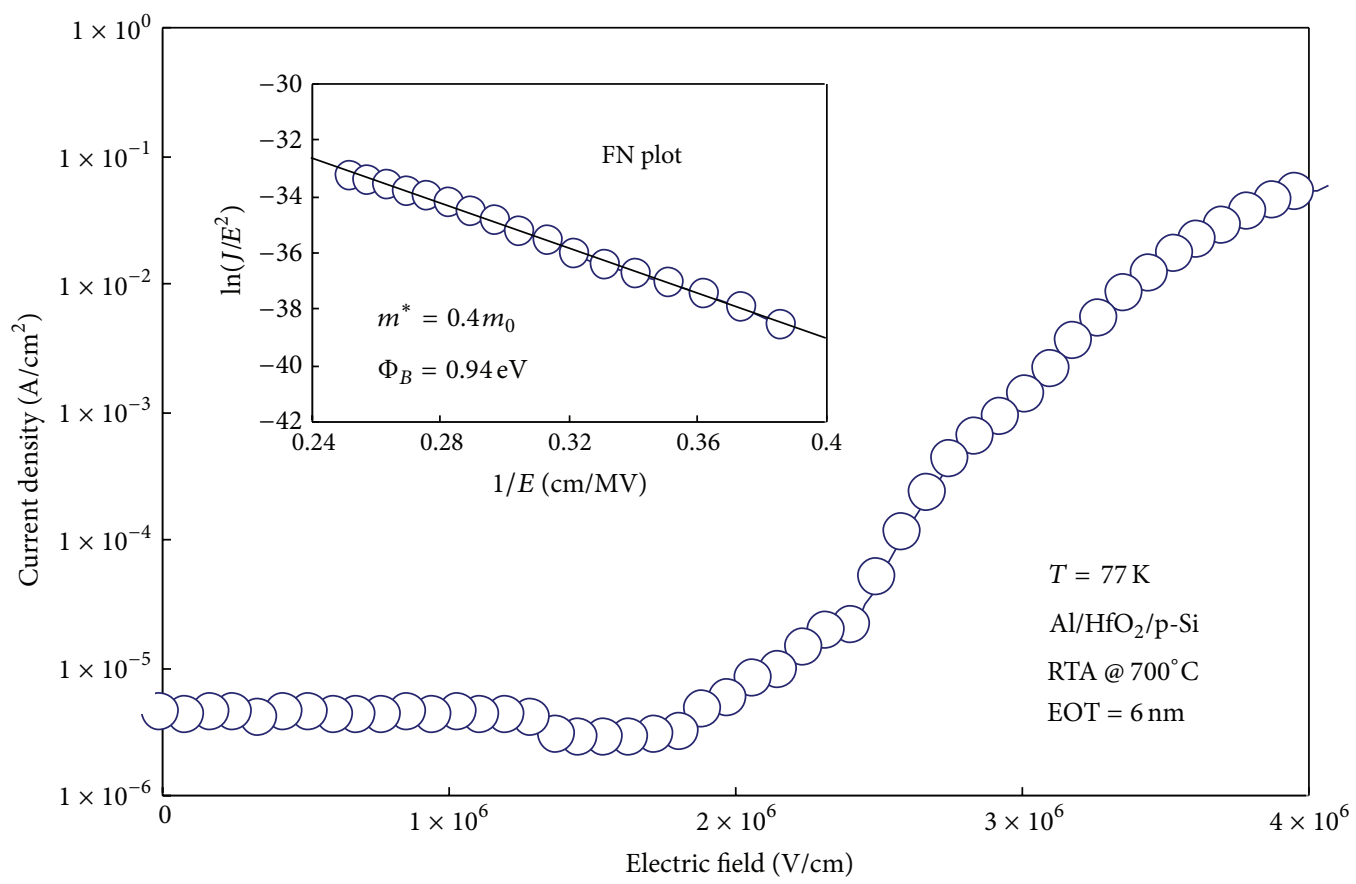

Figure 6: Characteristics of $J-E$ plots for $\mathrm{HfO}_{2}$ MIS capacitor at $77 \mathrm{~K}$. The inset graph presents the Fowler-Nordheim tunneling.

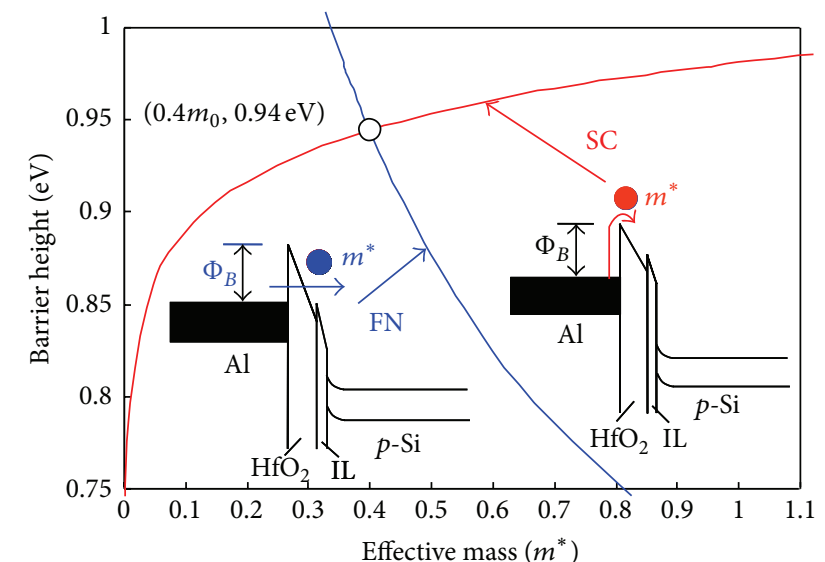

FIGURE 7: The extracted relationships between electron effective mass and $\mathrm{Al} / \mathrm{HfO}_{2}$ barrier height from the intercept of the Schottky plot at a high temperature $(465 \mathrm{~K})$ and the slope of the F-N plot at a low temperature $(77 \mathrm{~K})$. The band diagrams for the Schottky emission and F-N tunneling are also shown.

of the subthreshold carrier density with $V_{G}$ is indicated by the swing parameter $n_{\text {inv }}$, where $n_{\text {inv }}=S / v_{T}$ and $S$ are the subthreshold swing which is positive for NMOS and negative for PMOS. For EVB tunneling process, $N$ can be written as

$$
N=\frac{\varepsilon}{t} \cdot\left\{3 v_{T} \cdot \ln \left[1+\exp \left(\frac{q|V|-E_{g}}{3 k T}\right)\right]\right\}
$$

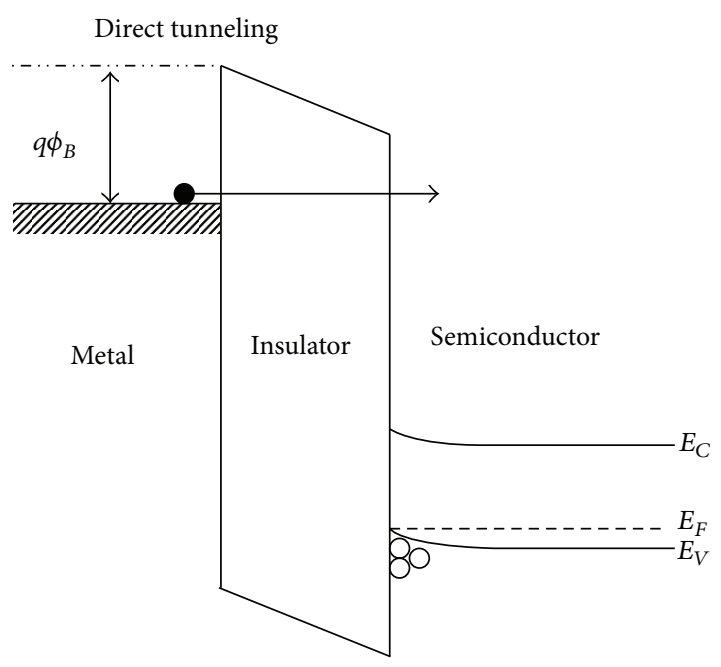

FIGURE 8: Schematic energy band diagram of direct tunneling in metal-insulator-semiconductor structure.

Equation (5) can be simplified using a binomial expansion and neglecting higher order terms, which leads to

$$
\begin{aligned}
J & \sim \exp \left\{-\frac{8 \pi\left(q \phi_{B}\right)^{3 / 2} \sqrt{2 m_{\mathrm{eff}}}}{3 h q|E|}\left[\frac{3|V|}{2 \phi_{B}}\right]\right\} \\
& \sim \exp \left\{-\frac{8 \pi \sqrt{2 q}}{3 h}\left(m_{\mathrm{eff}} \phi_{B}\right)^{1 / 2} \kappa \cdot t_{\mathrm{ox}, \mathrm{eq}}\right\} .
\end{aligned}
$$

Yeo et al. indicated the scaling limits of alternative gate dielectrics based on their direct tunneling characteristics and 


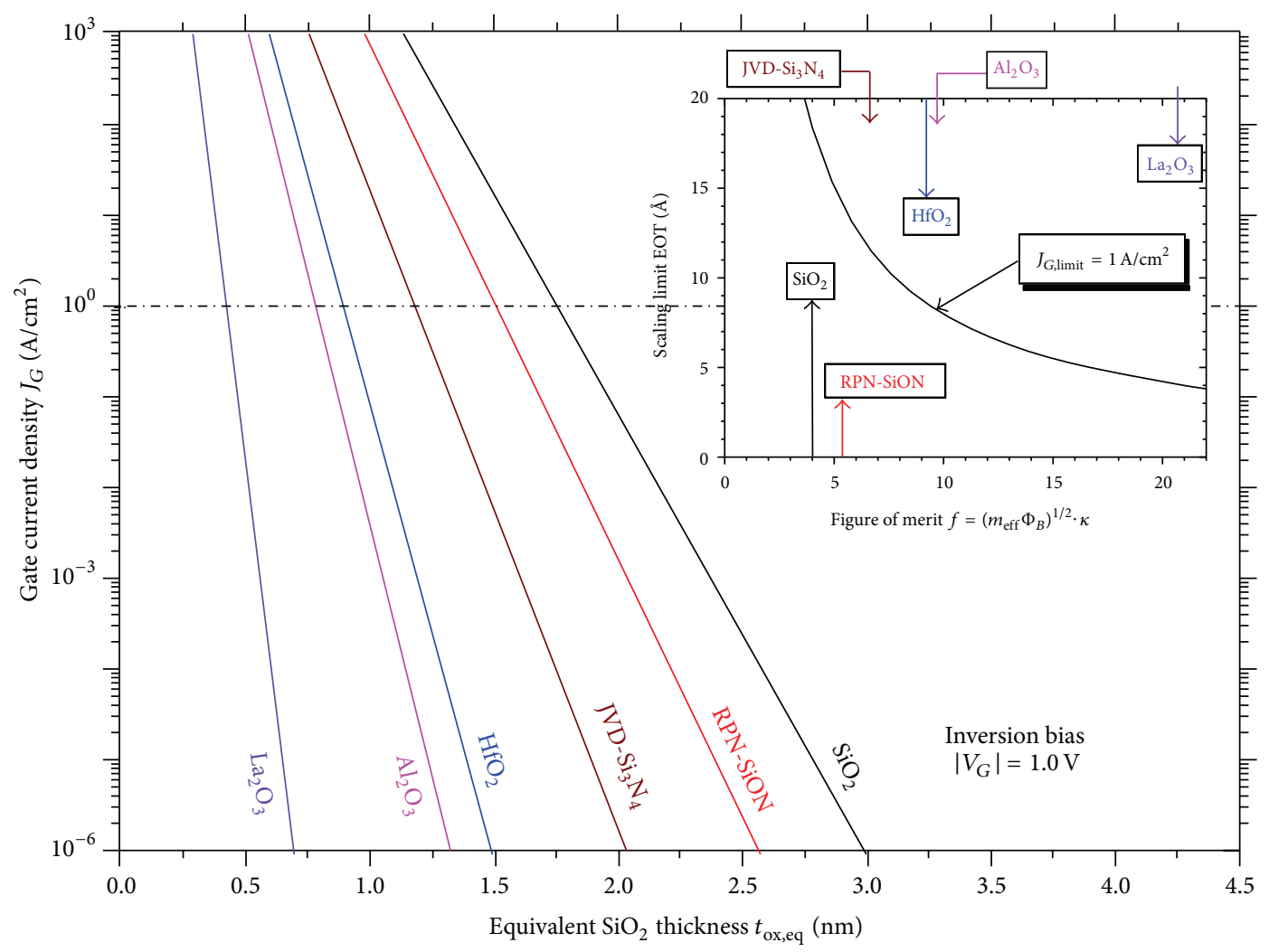

FIgURE 9: Scaling limit for several gate dielectrics when $V_{d d}=1.0 \mathrm{~V}$ and $J_{G, \text { limit }}=1 \mathrm{~A} / \mathrm{cm}^{2}[12]$.

gate leakage requirements for future CMOS technology [22]. The tunneling leakage current for a given EOT (equivalent oxide thickness, $\left.t_{\text {ox,eq }}\right)$ is not only dependent on the $\kappa$ value of a gate dielectric but also tunneling barrier height $\left(q \phi_{B}=\Phi_{B}\right)$ and tunneling effective mass $\left(m_{\text {eff }}\right)$. They introduced a figure of merit to compare the relative advantages of gate dielectric candidates. The figure of merit is given by $f=\left(m_{\mathrm{eff}} \Phi_{B}\right)^{1 / 2} \cdot \kappa$. Figure 9 shows the scaling limit for several gate dielectrics when $V_{G}$ or $V_{d d}$ is specified to be $1.0 \mathrm{~V}$ and the maximum tolerable gate current density $J_{G \text {,limit }}$ is $1 \mathrm{~A} / \mathrm{cm}^{2}$. A dielectric with a larger figure of merit possesses a lower scaling limit EOT, as shown in the inset of Figure 9. The EOT scaling limits of $\mathrm{La}_{2} \mathrm{O}_{3}$ and $\mathrm{HfO}_{2}$ are about $4 \AA$ and $9 \AA$, respectively.

2.4. Thermionic-Field Emission. Thermionic-field emission takes place intermediately between field emission and Schottky emission. In this condition, the tunneling electrons should have the energy between the Fermi level of metal and the conduction band edge of dielectric. The schematic energy band diagram of thermionic-field emission is shown in Figure 10(a). The difference between thermionic emission, thermionic-field emission, and field emission is shown in Figure 10 (b). The current density due to thermionic-field emission can be roughly expressed as [8]

$$
J=\frac{q^{2} \sqrt{m}(k T)^{1 / 2} E}{8 \hbar^{2} \pi^{5 / 2}} \exp \left(-\frac{q \phi_{B}}{k T}\right) \exp \left[\frac{\hbar^{2} q^{2} E^{2}}{24 m(k T)^{3}}\right] \text {. }
$$

\section{Bulk-Limited Conduction Mechanisms}

The bulk-limited conduction mechanisms depend on the electrical properties of the dielectric itself. The most important parameter in this type of conduction mechanism is the trap energy level in the dielectric films. The bulk-limited conduction mechanisms include (1) Poole-Frenkel emission, (2) hopping conduction, (3) ohmic conduction, (4) spacecharge-limited conduction, (5) ionic conduction, and (6) grain-boundary-limited conduction. Based on the bulklimited conduction mechanisms, some important electrical properties in the dielectric films can be extracted, including the trap energy level, the trap spacing, the trap density, the electronic drift mobility, and the dielectric relaxation time, the density of states in the conduction band.

3.1. Poole-Frenkel Emission. Poole-Frenkel (P-F) emission involves a mechanism which is very similar to Schottky emission; namely, the thermal excitation of electrons may emit from traps into the conduction band of the dielectric. Therefore, P-F emission is sometimes called the internal Schottky emission. Considering an electron in a trapping center, the Coulomb potential energy of the electron can be reduced by an applied electric field across the dielectric film. The reduction in potential energy may increase the probability of an electron being thermally excited out of the trap into the conduction band of the dielectric. The schematic energy band diagram of P-F emission is shown in Figure 11. For 
Thermionic-field emission

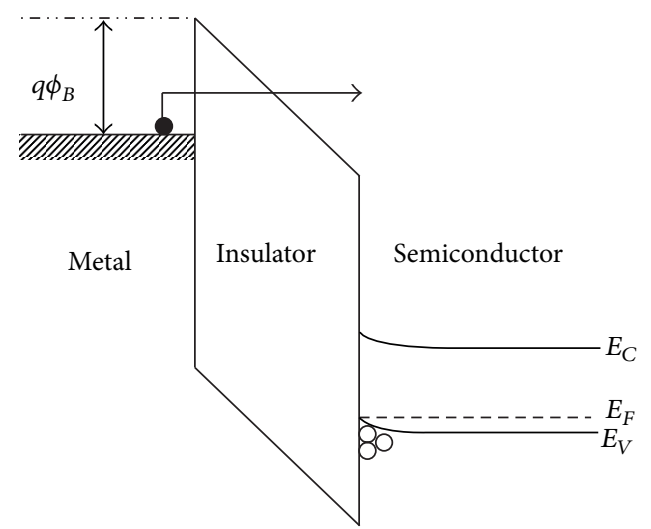

(a)

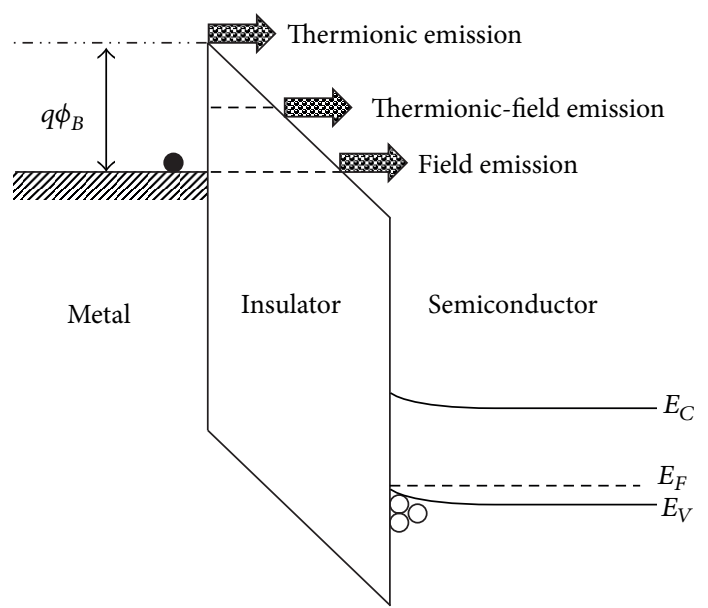

(b)

Figure 10: (a) Schematic energy band diagram of thermionic-field emission in metal-insulator-semiconductor structure. (b) Comparison of thermionic-field emission, thermionic emission, and field emission.

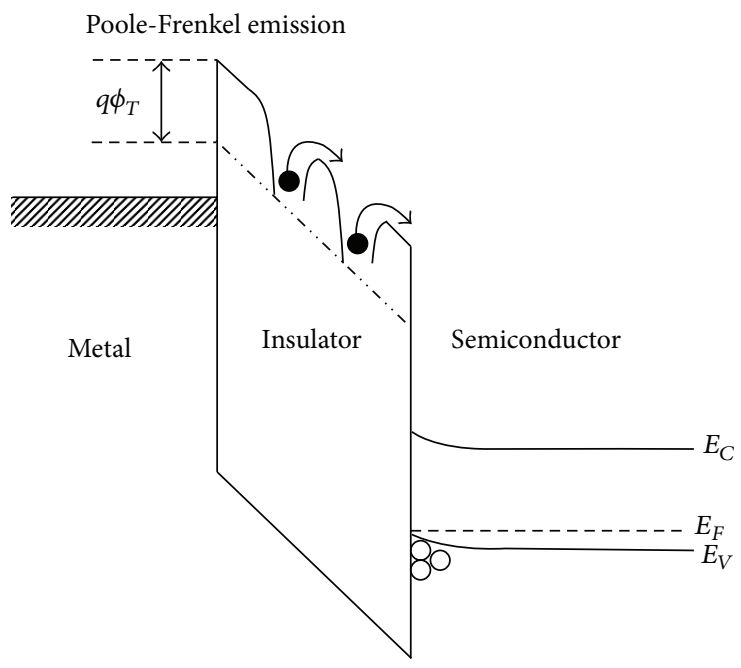

FIgURE 11: Schematic energy band diagram of Poole-Frenkel emission in metal-insulator-semiconductor structure.

a Coulombic attraction potential between electrons and traps, the current density due to the P-F emission is

$$
J=q \mu N_{C} E \exp \left[\frac{-q\left(\phi_{T}-\sqrt{q E / \pi \varepsilon_{i} \varepsilon_{0}}\right)}{k T}\right] \text {, }
$$

where $\mu$ is the electronic drift mobility, $N_{C}$ is the density of states in the conduction band, $q \phi_{T}\left(=\Phi_{T}\right)$ is the trap energy level, and the other notations are the same as defined before. Since P-F emission is owing to the thermal activation under an electric field, this conduction mechanism is often observed at high temperature and high electric field. Chiu et al. reported that the dominant conduction mechanism through $\mathrm{Pr}_{2} \mathrm{O}_{3}$ is the P-F emission at high temperature and high electric field [23]. Figure 12 shows the measured $J-E$ data and

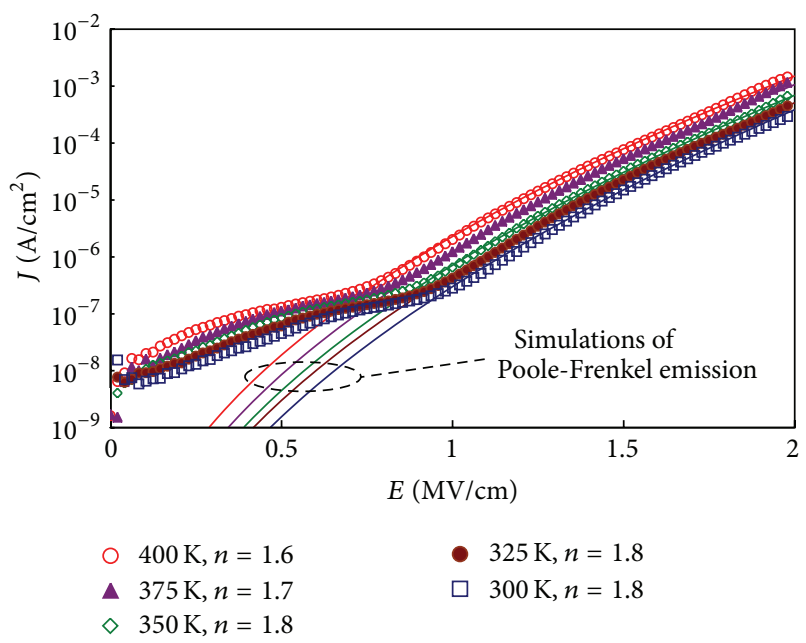

Figure 12: Characteristics of $J-E$ plot and simulation of P-F emission for the laminated $\operatorname{Pr}_{2} \mathrm{O}_{3} / \mathrm{SiON}$ MIS capacitors at high fields.

the modeled P-F emission curves for the laminated $\mathrm{Pr}_{2} \mathrm{O}_{3} /$ $\mathrm{SiON}$ MIS capacitors. The experimental data match the modeled curves very well in high electric fields $(>1 \mathrm{MV} / \mathrm{cm})$ from $300 \mathrm{~K}$ to $400 \mathrm{~K}$. Therefore, the dominant conduction mechanism is the P-F emission in high electric fields at temperatures ranging from $300 \mathrm{~K}$ to $400 \mathrm{~K}$. In addition, the trap energy level in $\mathrm{Pr}_{2} \mathrm{O}_{3}$ can be extracted by using the Arrhenius plot, as shown in Figure 13. The determined trap energy level in $\mathrm{Pr}_{2} \mathrm{O}_{3}$ is about $0.56 \pm 0.01 \mathrm{eV}$. Besides the simulation method, P-F plot is also a popular way to identify the trap energy level in dielectric films. For the P-F emission, a plot of $\ln (J / E)$ versus $E^{1 / 2}$ is linear. The trap barrier height can be extracted from the intercept of P-F plot. Figure 14 shows the P-F plot for a $17.4 \mathrm{~nm} \mathrm{ZrO}_{2}$ MIS capacitor in 


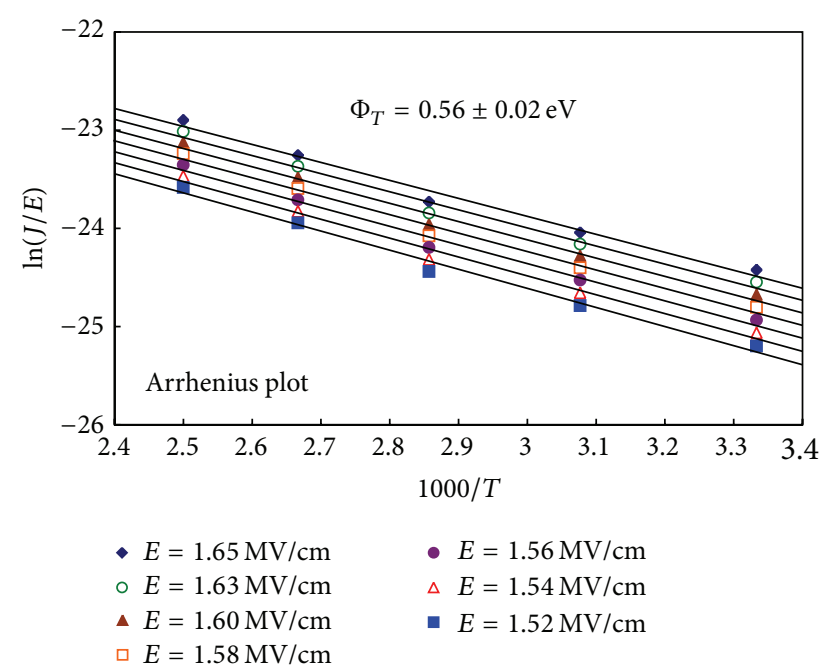

Figure 13: Arrhenius plot of the P-F emission for the laminated $\mathrm{Pr}_{2} \mathrm{O}_{3} / \mathrm{SiON}$ MIS capacitors at high fields.

the accumulation mode at high temperatures $(>425 \mathrm{~K})$ and in low electric fields $(<0.6 \mathrm{MV} / \mathrm{cm})$ [13]. Under the constraint of $n=\varepsilon_{r}^{1 / 2}$, the trap barrier height in $\mathrm{ZrO}_{2}$ films was extracted to be about $1.1 \mathrm{eV}$ according to the intercepts of the fitted lines in the P-F plot.

Aside from the intercept of P-F plot for the trap barrier, the slope of P-F plot is an important factor for determining the optical dielectric constant $\left(\varepsilon_{r}\right)$ in dielectric films. For the case of $\mathrm{Pr}_{2} \mathrm{O}_{3}$ MIS capacitors [23], the fitted $\varepsilon_{r}$ is $3 \pm 0.3$ and the fitted refractive index is $1.7 \pm 0.1$. The refractive index obtained by the electrical method is fairly close to the one obtained by the optical method ( $n=1.7-1.8)$. This implies that during the emission process, the electron transit time from the trap site to the barrier maximum position is shorter than the dielectric relaxation time. Consequently, the dielectric film does not have enough time to be polarized during the emission process and the optical dielectric constant should be used in this case. Besides the trap energy level and the optical dielectric constant, the electronic drift mobility in dielectric films can also be extracted by the analyses of $\mathrm{P}-\mathrm{F}$ emission. One can find the result of electron mobility in $\mathrm{Pr}_{2} \mathrm{O}_{3}$ films in [23]. Note that Angle and Talley proposed that the free electrons in P-F emission are emitted from the donor centers [24]. If there are other defect states such as trap states or acceptor centers in dielectric films, the number of emitted free electrons from the donor center will decrease. The existence of the trapping centers may influence the relation of current density and electric field. Accordingly, the P-F emission is dependent on the concentration of trap centers $\left(N_{t}\right)$ and donor centers $\left(N_{d}\right)$. When $N_{t}<N_{d}$, the conduction mechanism is called the normal P-F emission [24]. When $N_{t} \cong N_{d}$, the conduction mechanism is called the modified P-F emission or anomalous Poole-Frenkel effect [24]. In such a case, the slope of P-F plot is reduced by half and equals the slope of Schottky plot. To distinguish the Schottky emission and modified P-F emission, the effect of different electrode materials on the conduction characteristics is a valuable means.

3.2. Hopping Conduction. Hopping conduction is due to the tunneling effect of trapped electrons "hopping" from one trap site to another in dielectric films. Figure 15 shows the schematic energy band diagram of hopping conduction. The expression of hopping conduction is $[5,10,23]$

$$
J=q a n v \exp \left[\frac{q a E}{k T}-\frac{E_{a}}{k T}\right]
$$

where $a$ is the mean hopping distance (i.e., the mean spacing between trap sites), $n$ is the electron concentration in the conduction band of the dielectric, $v$ is the frequency of thermal vibration of electrons at trap sites, and $E_{a}$ is the activation energy, namely, the energy level from the trap states to the bottom of conduction band $\left(E_{C}\right)$; the other terms are as defined above. The P-F emission corresponds to the thermionic effect and the hopping conduction corresponds to the tunnel effect. In P-F emission, the carriers can overcome the trap barrier through the thermionic mechanism. However, in hopping conduction, the carrier energy is lower than the maximum energy of the potential barrier between two trapping sites. In such case, the carriers can still transit using the tunnel mechanism.

Chiu et al. [23] reported that the experimental $J-E$ data match the simulated hopping conduction curves very well from $300 \mathrm{~K}$ to $400 \mathrm{~K}$ in low electric fields $(<0.6 \mathrm{MV} / \mathrm{cm})$ in a $\mathrm{Pr}_{2} \mathrm{O}_{3}$ MIS structure, as shown in Figure 16. From the simulations of hopping conduction, the mean hopping distance in the $\operatorname{Pr}_{2} \mathrm{O}_{3}$ films was determined to be about $1.5 \pm$ $0.1 \mathrm{~nm}$. Using the slopes of Arrhenius plot in low fields, the activation energy was determined to be about $50 \pm 1 \mathrm{meV}$, as shown in Figure 17. Furthermore, according to (12), the trap spacing can be extracted by the slope of linear part of $\log (J)$ versus $E$. Figure 18 shows the $J-E$ characteristics at temperatures ranging from $300 \mathrm{~K}$ to $425 \mathrm{~K}$ for a $\mathrm{Pt} / \mathrm{MgO} / \mathrm{Pt}$ memory device in high resistance state [25]. Simulation results show that the measured data match the theory of hopping conduction very well when the electric field is larger than about $0.25 \mathrm{MV} / \mathrm{cm}$. Based on the slope in Figure 18, the trap spacing in $\mathrm{MgO}$ can be determined to be about $1.0 \mathrm{~nm}$ [25]. In Figure 18, we can observe an interesting characteristic, that is, a lower current density is observed at a higher temperature. This finding is far different from the normal $J-E$ characteristics in dielectric films in which the higher current density can be achieved in a higher temperature. In the case of $\mathrm{Pt} / \mathrm{MgO} / \mathrm{Pt}$, a simulation work can be adopted by varying the trapping level [25]. Therefore, the temperature dependence of the trap energy levels in $\mathrm{MgO}$ can be obtained, as shown in Figure 19. The trap energy level increases with increasing temperature. This result indicates that the defects with deeper level are activated by the elevated temperature. Hence, the deeper trap level activated at higher temperature leads to the exponential decrease in current density.

3.3. Ohmic Conduction. Ohmic conduction is caused by the movement of mobile electrons in the conduction band and 


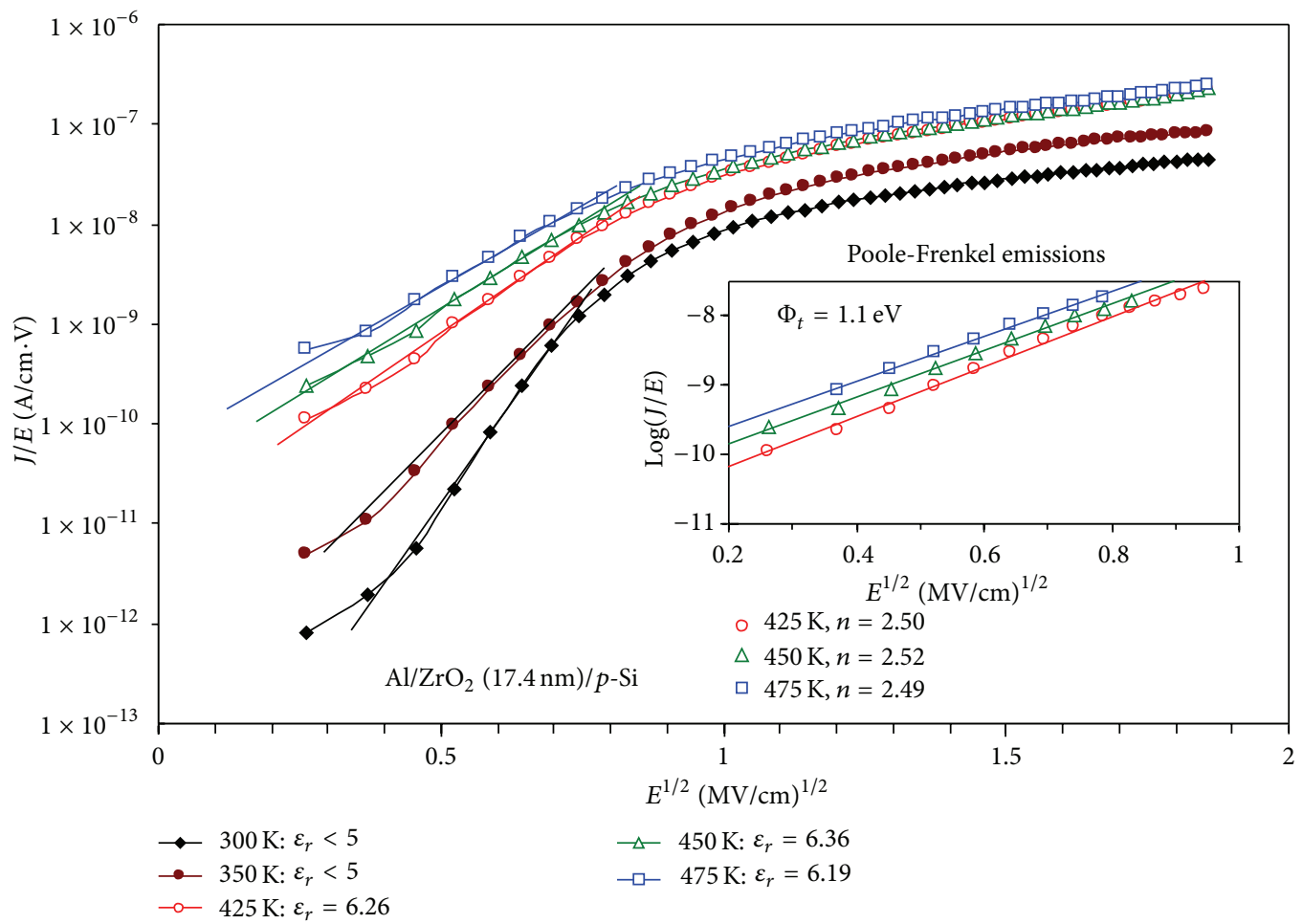

FIGURE 14: Characteristics of P-F emission at various temperatures. Inset graph presents Poole-Frenkel emission constrained by $n=\varepsilon_{r}^{1 / 2}$ at high temperatures.

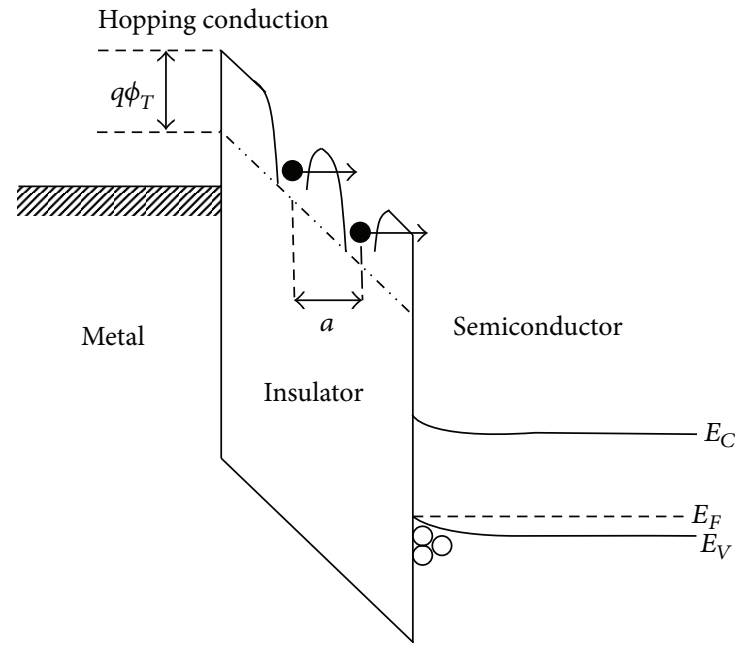

FIGURE 15: Energy band diagram of hopping conduction in metalinsulator-semiconductor structure.

holes in the valence band. In this conduction mechanism, a linear relationship exists between the current density and the electric field. Figure 20 shows a schematic energy band diagram of the Ohmic conduction due to electrons. Although the energy band gap of dielectrics is by definition large, there will still be a small number of carriers that may be generated due to the thermal excitation. For example, the electrons may be excited to the conduction band, either from the valence

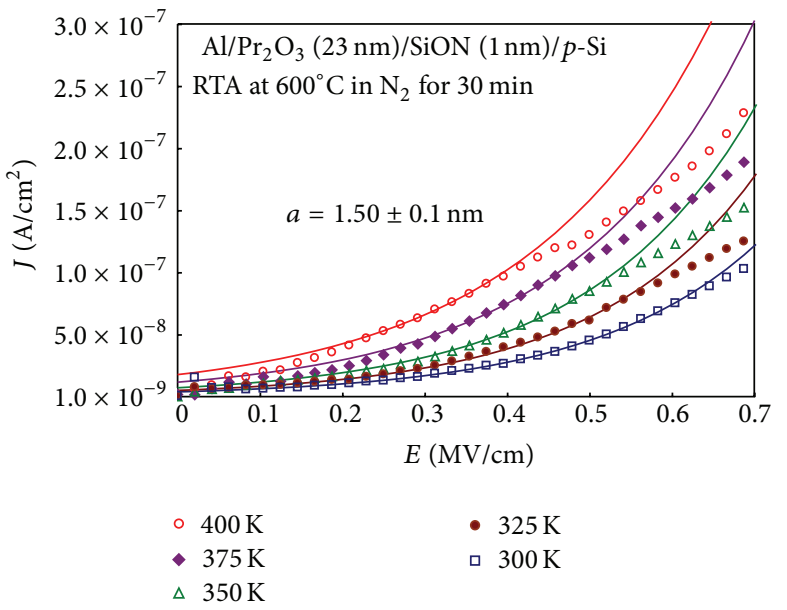

FIGURE 16: $J$-E characteristics and simulation of hopping conduction for a laminated $\mathrm{Pr}_{2} \mathrm{O}_{3} / \mathrm{SiON}$ MIS capacitors at low electric fields.

band or from the impurity level. The carrier numbers will be very small but they are not zero. The current density of ohmic conduction can be expressed as

$$
J=\sigma E=n q \mu E, \quad n=N_{C} \exp \left[\frac{-\left(E_{C}-E_{F}\right)}{k T}\right],
$$

where $\sigma$ is electrical conductivity, $n$ is the number of electrons in the conduction band, $\mu$ is electron mobility, and $N_{C}$ is 


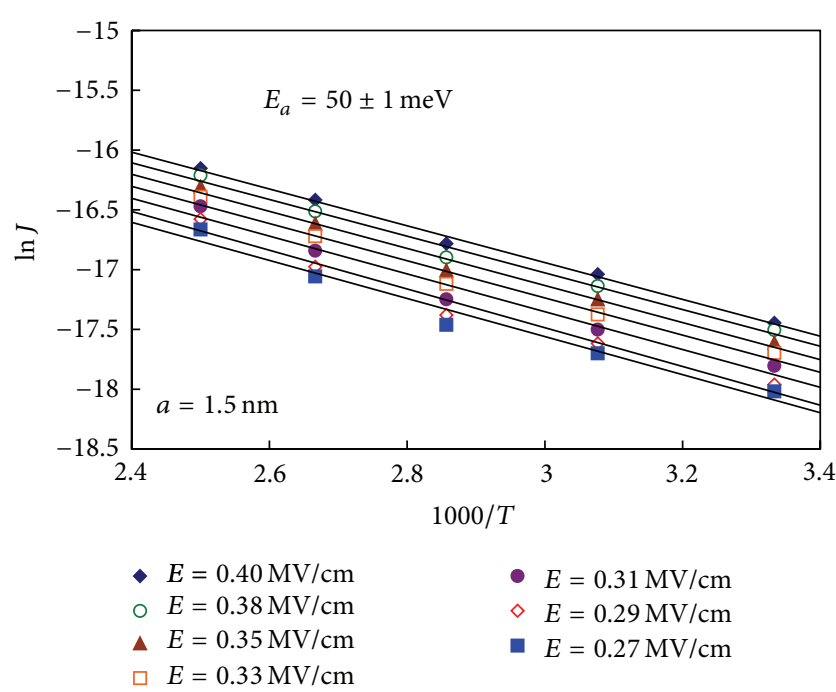

Figure 17: Arrhenius plot of the hopping conduction at low fields for the laminated $\mathrm{Pr}_{2} \mathrm{O}_{3} / \mathrm{SiON}$ MIS capacitors.

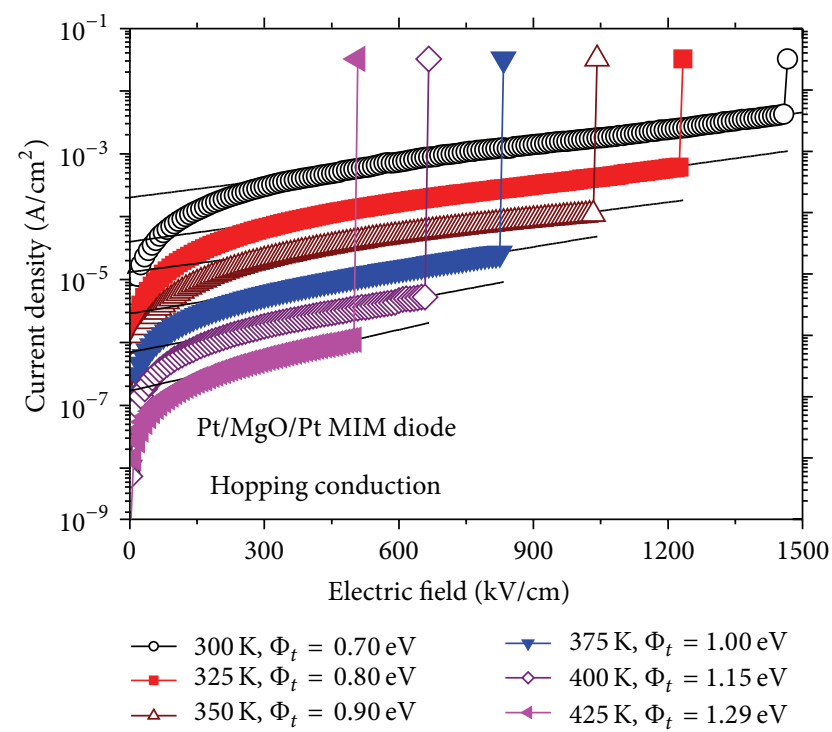

FIGURE 18: Experimental data and simulation curves of hopping conduction in high resistance state in $\mathrm{Pt} / \mathrm{MgO} / \mathrm{Pt}$ memory device.

the effective density of states of the conduction band; the other terms are as defined above.

Because the energy band gap of a dielectric is very large, we can assume that the Fermi level $E_{F}$ is close to the middle of the energy band gap; that is, $E_{C}-E_{F} \sim E_{g} / 2$. In this case, the ohmic conduction current is $J=q \mu E N_{C} \exp \left(-E_{g} / 2 k T\right)$. The magnitude of this current is very small. This current mechanism may be observed if there is no significant contribution from other conduction mechanisms of current transport in dielectrics [10]. The ohmic conduction current due to mobile electrons in the conduction band or similarly holes in the valence band is linearly dependent on the electric field. This current usually may be observed at very low voltage in the current-voltage $(I-V)$ characteristics of the dielectric films.

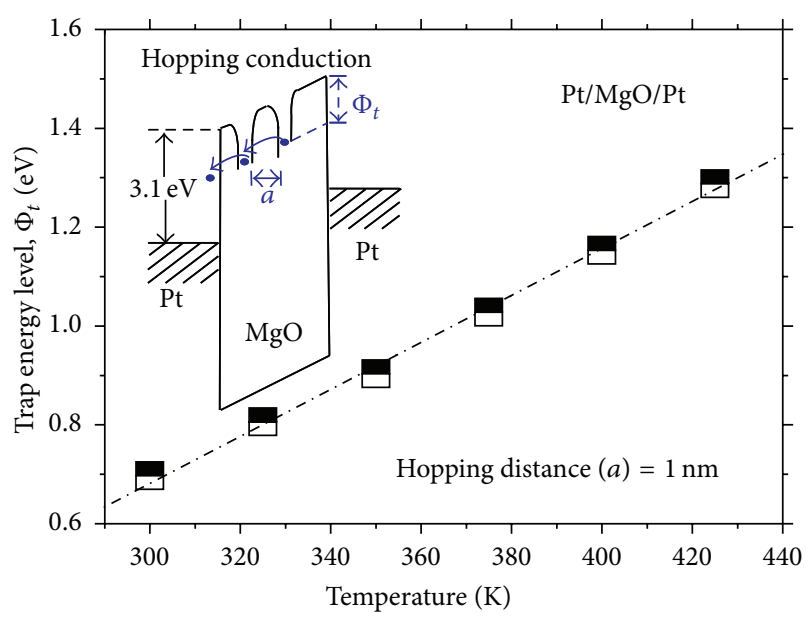

FIGURE 19: Temperature dependence of the trap energy levels in high resistance state. Inset graph shows the band diagram of hopping conduction in $\mathrm{Pt} / \mathrm{MgO} / \mathrm{Pt}$ memory cells.

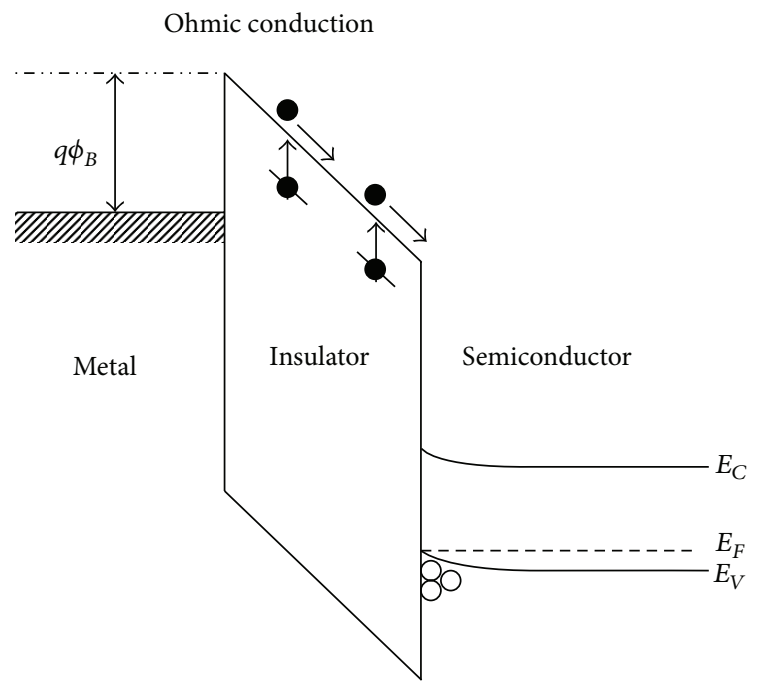

FIgURE 20: Energy band diagram of ohmic conduction in metalinsulator-semiconductor structure.

Recently, the resistance switching behaviors in dielectric films have been extensively studied [26-29]. The ohmic conduction can be often observed in low resistance state in the resistance switching memory devices. Figure 21 shows the $J-E$ curves in a double-logarithmic plot in low resistance state in $\mathrm{Pt} / \mathrm{ZnO} / \mathrm{Pt}$ memory devices [29]. The linear relation between current density and electric field is observed, which matches the ohmic conduction very well because the slopes are very close to 1. In Figure 21, the current density increases with increasing temperature. Hence, the linear relation between electrical conductivity $(\sigma)$ and inverse temperature can be derived from Figure 21, as shown in Figure 22. Using the Arrhenius plot, the Fermi level $\left(E_{F}\right)$ of $\mathrm{ZnO}$ is determined to be about $0.4 \mathrm{eV}$ below the conduction band edge in $\mathrm{ZnO}$ $\left(E_{C}\right)$, as shown in the inset of Figure 22. Consequently, the product of $\mu$ and $N_{C}$ at each temperature can be extracted by 


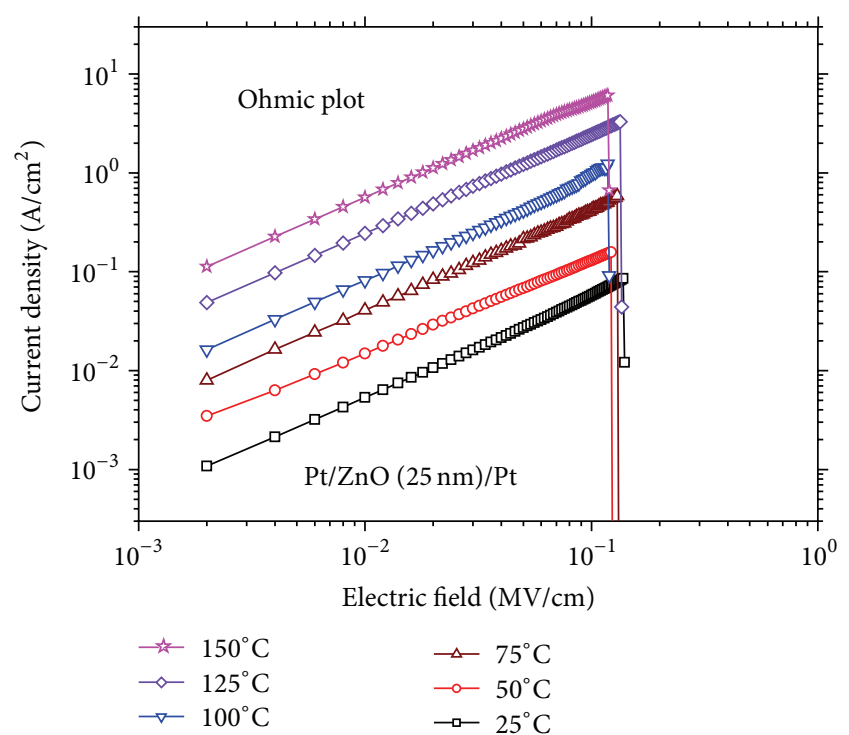

FIGURE 21: Linear relation between current density and electric field at temperature ranging from 25 to $150^{\circ} \mathrm{C}$ in low resistance state in $\mathrm{Pt} / \mathrm{ZnO} / \mathrm{Pt}$ memory devices.

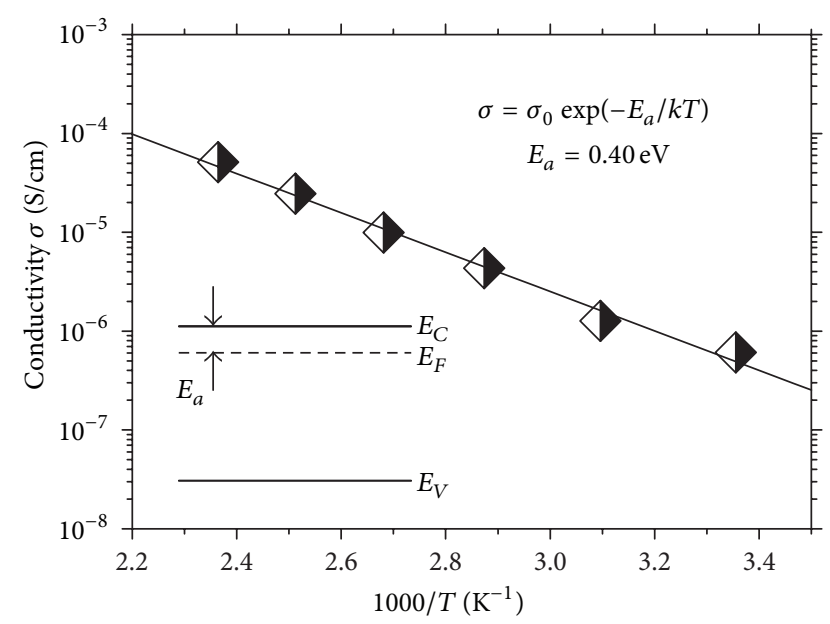

FIGURE 22: Temperature dependence of the electrical conductivity in low resistance state in $\mathrm{Pt} / \mathrm{ZnO} / \mathrm{Pt}$ memory devices. The inset graph shows the location of Fermi level in ohmic conduction.

the combination of $E_{F}$ and $\sigma$. In addition, $N_{C}$ is a function of temperature, which is proportional to $\beta T^{3 / 2}$, where $\beta$ is a constant [30]. The effective density of states of the conduction band $\left(N_{C}\right)$ in $\mathrm{ZnO}$ at room temperature is $4.8 \times 10^{18} \mathrm{~cm}^{-3}$ [31]. Therefore, the temperature-dependent electron mobility $(\mu)$ and effective density of states of the conduction band $\left(N_{C}\right)$ in $\mathrm{ZnO}$ can be obtained, as shown in Figure 23. At room temperature the electron mobility in $\mathrm{ZnO}$ films is about $4.6 \mathrm{~cm}^{2} / \mathrm{V}$-s.

3.4. Space-Charge-Limited Conduction. The mechanism of space-charge-limited conduction (SCLC) is similar to the transport conduction of electrons in a vacuum diode.
The thermionic cathode of a vacuum diode can emit electrons with a Maxwellian distribution of initial velocities $(v)$. The corresponding charge distribution can be written by the Poisson's equation:

$$
\frac{\partial^{2} V}{\partial x^{2}}=-\frac{\rho(x)}{\varepsilon_{0}} .
$$

Moreover, in the steady state, with the condition $v(x)=$ $[2 q V(x) / m]^{1 / 2}$, the continuity equation is

$$
j_{x}=q n(x) v(x) .
$$

The current density-voltage $(J-V)$ characteristic of a vacuum diode is governed by the Child's law:

$$
J=\frac{4 \varepsilon_{0}}{9}\left(\frac{2 e}{m}\right)^{1 / 2} \frac{V^{3 / 2}}{d^{2}}
$$

In a solid material, the space-charge-limited current is caused by the injection of electrons at an ohmic contact. The continuity equation should include the diffusion component and can be written as

$$
j_{x}=e n(x) v(x)+e D \frac{d n}{d x} .
$$

A typical $J-V$ characteristic plotted in a log-log curve for space-charge-limited current is shown in Figure 24. The $J-V$ characteristic in the $\log J-\log V$ plane is bounded by the three limited curves, namely, ohm's law $\left(J_{\mathrm{Ohm}} \propto V\right)$, traps-filled limit (TFL) current $\left(J_{\mathrm{TFL}} \propto V^{2}\right)$, and Child's law ( $J_{\text {Child }} \propto$ $\left.V^{2}\right) . V_{\text {tr }}$ and $V_{\text {TFL }}$ are the transition voltage at the departure from ohm's law and TFL curve, respectively:

$$
\begin{gathered}
J_{\mathrm{Ohm}}=q n_{0} \mu \frac{V}{d}, \\
J_{\mathrm{TFL}}=\frac{9}{8} \mu \varepsilon \theta \frac{V^{2}}{d^{3}}, \\
J_{\text {Child }}=\frac{9}{8} \mu \varepsilon \frac{V^{2}}{d^{3}}, \\
V_{\mathrm{tr}}=\frac{8}{9} \times \frac{q n_{0} d^{2}}{\varepsilon \theta}, \\
\theta=\frac{N_{\mathrm{C}}}{g_{n} N_{t}} \exp \left(\frac{E_{t}-E_{C}}{k T}\right), \\
V_{\mathrm{TFL}}=\frac{q N_{t} d^{2}}{2 \varepsilon}, \\
\tau_{c}=\frac{d^{2}}{\mu \theta V_{\mathrm{tr}}}, \\
\tau_{d}=\frac{\varepsilon}{q n \mu \theta},
\end{gathered}
$$

where $n_{o}$ is the concentration of the free charge carriers in thermal equilibrium, $V$ is the applied voltage, $d$ is the thickness of thin films, $\varepsilon$ is the static dielectric constant, $\theta$ is the 


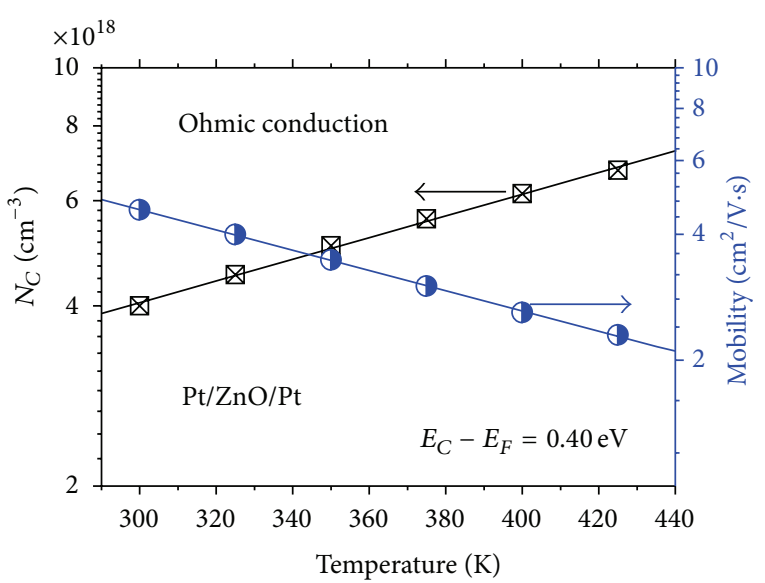

FIgURE 23: Temperature dependence of the electron mobility and the effective density of states of the conduction band in low resistance state in $\mathrm{Pt} / \mathrm{ZnO} / \mathrm{Pt}$ memory devices.

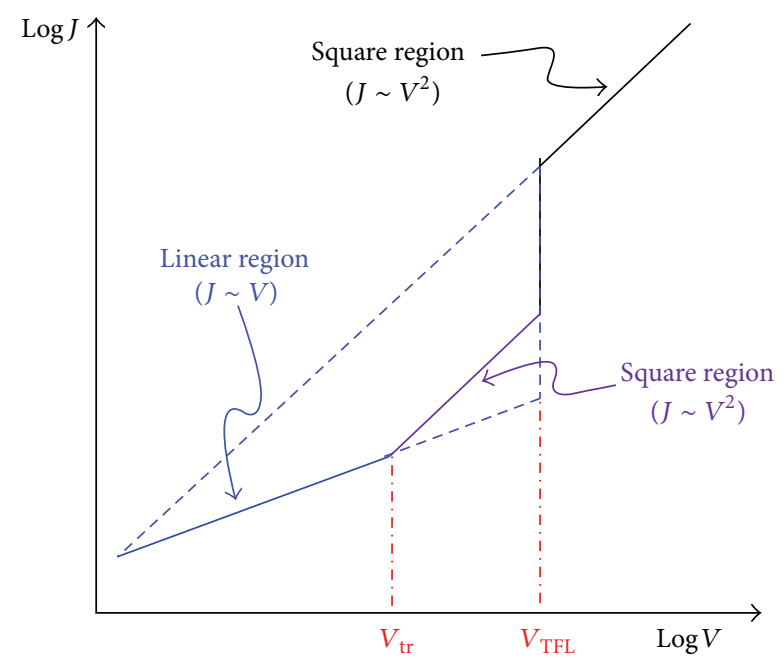

FIgURe 24: A typical current density-voltage $(I-V)$ characteristic of space-charge-limited conduction current. $V_{\text {tr }}$ is transition voltage. $V_{\text {TFL }}$ is trap-filled limit voltage.

ratio of the free carrier density to total carrier (free and trapped) density, $g_{n}$ is the degeneracy of the energy state in the conduction band, $E_{t}$ is the trap energy level, $N_{t}$ is the trap density, $n$ is the concentration of the free carrier in the insulator, and the other terms are as defined above. It is noticed that (20) is the Mott-Gurney relation which indicates the space-charge-limited current under the condition of single type of carriers and no traps. If there are traps in the dielectric, the SCL conduction can be described by (19) based on the assumption of monoenergetical trapping levels in the dielectric $[5-7,10]$.

At low applied voltages $\left(V<V_{\text {tr }}\right), J-V$ characteristics followed the ohm's law, which implies that the density of thermally generated free carriers $\left(n_{0}\right)$ inside the films is larger than the injected carriers [32]. This ohmic mode takes place in the electrically quasineutral state corresponding to the situation when partial trap centers are filled at weak injection. When the transition from the ohmic to the space-chargelimited region, the carrier transit time $\left(\tau_{c}\right)$ at $V_{\text {tr }}$ (the minimum voltage required for the transition) becomes equal to the dielectric relaxation time $\left(\tau_{d}\right)$ [2]. The onset of the departure from ohm's law or the onset of the SCL conduction takes place when the applied voltage reaches the value of $V_{\text {tr }}$. Accordingly, $\tau_{c} \cong \tau_{d}$ can be extracted at the transition point $V_{\mathrm{tr}}$. If the applied voltage $V$ is smaller than $V_{\mathrm{tr}}$, then the carrier transit time $\tau_{c}$ is larger than the dielectric relaxation time $\tau_{d}$. This implies that the injected carrier density $n$ is small in comparison with $n_{0}$ and that the injected carriers will redistribute themselves with a tendency to maintain electric charge neutrality internally in a time comparable to $\tau_{d}$. Consequently, the injected carriers have no chance to travel across the insulator. The redistribution of the charge is known as dielectric relaxation. The ohmic behavior can be observed only after these space charge carriers become trapped. Figure 25 shows the schematic diagrams of carrier distributions in dielectric film in SCLC mechanism under the conditions of (a) very weak injection $\left(V<V_{\text {tr }}, n<n_{0}\right.$, $\tau_{c}>\tau_{d}$ ), (b) dielectric relaxation and carriers redistribution, and (c) weak injection at $V_{\mathrm{tr}}\left(V=V_{\mathrm{tr}}, n=n_{0}, \tau_{c}=\tau_{d}\right)$.

In the case of strong injection, the traps are filled up and a space charge appears. When $V>V_{\operatorname{tr}}$ and $\tau_{c} \sim \tau_{d}$ or $\tau_{c}<\tau_{d}$, the injected excess carriers dominate the thermally generated carrier since the injected carrier transit time is too short for their charge to be relaxed by the thermally generated carriers. It is noted that for $V<V_{\operatorname{tr}}, \tau_{c}$ increases with decreasing $V$ but $\tau_{d}$ remains almost constant, while for $V>V_{\mathrm{tr}}, \tau_{c}$ decreases with increasing $V$ and $\tau_{d}$ also decreases with increasing $V$ since the increase in $V$ causes an increase in free carrier density in the dielectric. The increase of applied voltage may increase the density of free carriers resulting from injection to such a value that the Fermi level $\left(E_{F n}\right)$ moves up above the electron trapping level $\left(E_{t}\right)$. The trap-filled limit (TFL) is the condition for the transition from the trapped $J-V$ characteristics to the trap-free $J-V$ characteristics. It can be imagined that after all traps are filled up, the subsequently injected carriers will be free to move in the dielectric films, so that at the subthreshold voltage $\left(V_{\mathrm{TFL}}\right)$ to set on this transition, the current will rapidly jump from its low traplimited value to a high trap-free SCL current. $V_{\mathrm{TFL}}$ is defined as the voltage required to fill the traps or, in other words, as the voltage at which Fermi level $\left(E_{F n}\right)$ passes through $E_{t}$.

In the case of very strong injection, all traps are filled and the conduction becomes the space-charge-limited (Child's law). Thus a space charge layer in the dielectric builds up and the electric field cannot be regarded as constant any longer. While the bias voltage reaches $V_{\mathrm{TFL}}$ in the strong injection mode, the traps get gradually saturated, which means that the Fermi-level gets closer to the bottom of the conduction band. This results in a strong increase of the number of free electrons, thus explaining the increase of the current for $V=$ $V_{\mathrm{TFL}}$. For the voltage $>V_{\mathrm{TFL}}$, the current is fully controlled by the space charge, which limits the further injection of free carriers in the dielectric. Square law dependence of 


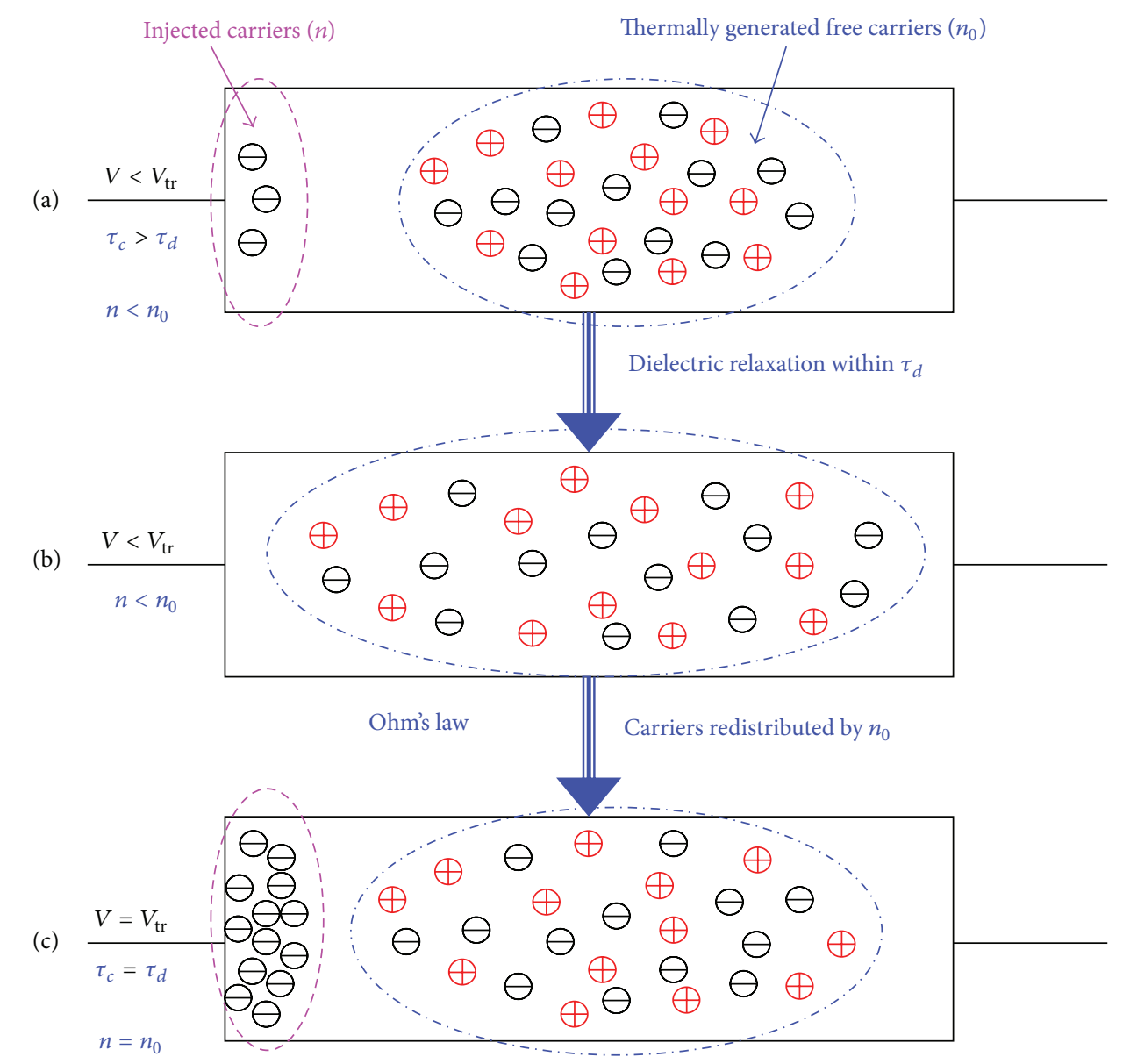

FIGURE 25: Carriers distribution in dielectric film under carrier weak injection $\left(V \leq V_{\mathrm{tr}}\right)$ in space-charge-limited conduction. (a) Very weak injection $\left(V<V_{\mathrm{tr}}, n<n_{0}, \tau_{c}>\tau_{d}\right)$, (b) dielectric relaxation and carriers redistribution, and (c) weak injection at $V_{\mathrm{tr}}\left(V=V_{\mathrm{tr}}, n=n_{0}, \tau_{c}=\tau_{d}\right)$. $V_{\mathrm{tr}}$ is the transition voltage at the onset of departure from ohm's law; $n$ is the concentration of injected carriers; $n_{0}$ is the concentration of thermally generated free carriers in dielectric film; $\tau_{c}$ is the carrier transit time; $\tau_{d}$ is the dielectric relaxation time.

the current $\left(J \sim V^{2}\right.$, Child's law) is the consequence of the space charge controlled current. Figure 26 shows the schematic diagrams of carrier and trap distributions in dielectric film in SCLC mechanism under the conditions of (a) strong injection $\left(V>V_{\mathrm{tr}}, n<n_{0}<N_{t}, \tau_{c}<\tau_{d}\right)$, (b) trap-filled-limited conduction $\left(V_{\mathrm{tr}}<V<V_{\mathrm{TFL}}, n<n_{0}<N_{t}\right.$, $\left.E_{F n}<E_{t}\right)$ : trapped behavior, parts of traps are filled up, and (c) space-charge-limited conduction $\left(V>V_{\mathrm{TFL}}, n_{0}>N_{t}\right.$, $E_{F n}>E_{t}$ ): trap-free behavior, all traps are filled up.

A report [32] showed that the dominant conduction mechanism through the polycrystalline $\mathrm{La}_{2} \mathrm{O}_{3}$ films is spacecharge-limited current, as shown in Figure 27. Three different regions, ohm's law region, trap-filled-limited region, and Child's law region are observed in the current density-voltage $(J-V)$ characteristics at room temperature. Based on the SCLC study in [32], some valuable electrical properties in polycrystalline $\mathrm{La}_{2} \mathrm{O}_{3}$ were obtained. For example, at room temperature, the trap density $\left(N_{t}\right)$ is about $9.2 \times 10^{17} \mathrm{~cm}^{-3}$, the trap energy level $\left(E_{t}\right)$ is about $0.21 \mathrm{eV}$, the trap capture cross-section $\left(\sigma_{t}\right)$ is about $1.2 \times 10^{-21} \mathrm{~cm}^{2}$, the effective density of states in the conduction band $\left(N_{C}\right)$ is about
$5.5 \times 10^{18} \mathrm{~cm}^{-3}$, the maximum of dielectric relaxation time $\left(\tau_{d, \max }\right)$ is about $8.8 \times 10^{-5} \mathrm{~s}$, and the electron mobility $(\mu)$ is about $8.2 \times 10^{-7} \mathrm{~cm}^{2} / \mathrm{V}$-s. In addition, some important electrical properties in polycrystalline $\mathrm{Dy}_{2} \mathrm{O}_{3}$ were also obtained according to the SCLC mechanism [33]. At $350 \mathrm{~K}$, the trap density $\left(N_{t}\right)$ is about $1.5 \times 10^{19} \mathrm{~cm}^{-3}$, the trap energy level $\left(E_{t}\right)$ is about $0.20 \mathrm{eV}$, the trap capture cross-section $\left(\sigma_{t}\right)$ is about $3.2 \times 10^{-21} \mathrm{~cm}^{2}$, the effective density of states in the conduction band $\left(N_{C}\right)$ is about $4.5 \times 10^{21} \mathrm{~cm}^{-3}$, the maximum of dielectric relaxation time $\left(\tau_{d, \max }\right)$ is about $8.2 \times 10^{-6} \mathrm{~s}$, and the electron mobility $(\mu)$ is about $1.2 \times$ $10^{-6} \mathrm{~cm}^{2} / \mathrm{V}$-s.

3.5. Ionic Conduction. Ionic conduction results from the movement of ions under an applied electric field. The movement of the ions may come from the existence of lattice defects in the dielectric films. Due to the influence of external electric field on defect energy level, the ions may jump over a potential barrier from one defect site to another. Figure 28(a) shows a schematic energy band diagram of ionic 
(a)

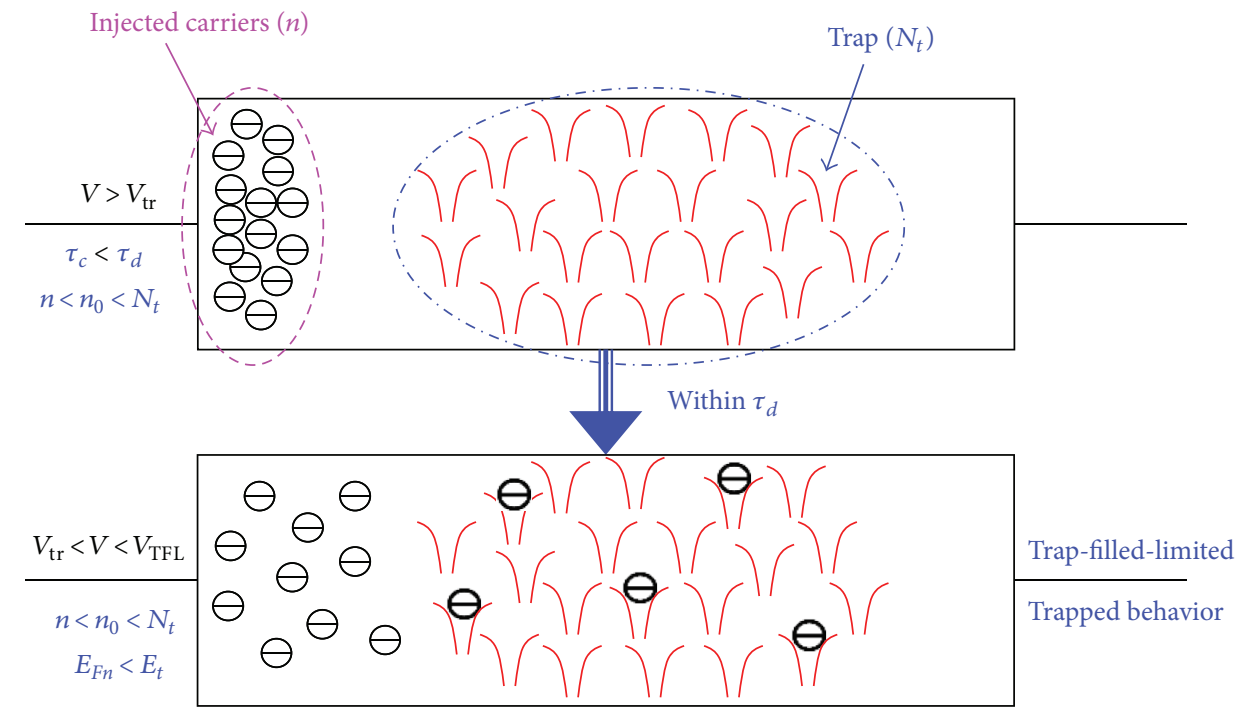

(c)

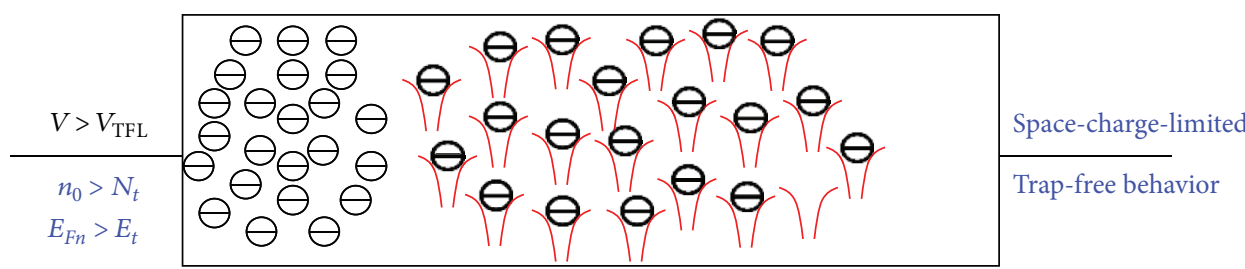

Figure 26: Carriers distribution in dielectric film under carrier strong injection $\left(V>V_{\text {tr }}\right)$ in space-charge-limited conduction. (a) Strong injection $\left(V>V_{\mathrm{tr}}, n<n_{0}<N_{t}, \tau_{c}<\tau_{d}\right)$, (b) trap-filled-limited $\left(V_{\mathrm{tr}}<V<V_{\mathrm{TFL}}, n<n_{0}<N_{t}, E_{F n}<E_{t}\right)$ : parts of traps are filled up (trapped behavior), and (c) space-charge-limited $\left(V>V_{\mathrm{TFL}}, n_{0}>N_{t}, E_{F n}>E_{t}\right)$ : all traps are filled up (trap-free behavior). $V_{\mathrm{tr}}$ and $V_{\mathrm{TFL}}$ are the transition voltage at the departure from ohm's law and TFL curve, respectively; $n$ is the concentration of injected carriers; $n_{0}$ is the concentration of thermally generated free carriers; $N_{t}$ is the trap density; $\tau_{c}$ is the carrier transit time; $\tau_{d}$ is the dielectric relaxation time; $E_{F n}$ is the Fermi level; $E_{t}$ is the trapping level.

conduction without the applied electric field. Figure 28(b) shows the condition with the applied electric field [10]. The ionic conduction current can be expressed as

$$
J=J_{0} \exp \left[-\left(\frac{q \phi_{B}}{k T}-\frac{E q d}{2 k T}\right)\right]
$$

where $J_{0}$ is the proportional constant, $q \phi_{B}$ is the potential barrier height, $E$ is the applied electric field, $d$ is the spacing of two nearby jumping sites, and the other terms are as defined above. Because the ion mass is large, the mechanism of ionic conduction is usually not important for the applications of dielectric films in CMOS technology.

3.6. Grain-Boundary-Limited Conduction. In a polycrystalline dielectric material, the resistivity of the grain boundaries may be much higher than that of the grains. Therefore, the conduction current could be limited by the electrical properties of the grain boundaries. In this case, the conduction mechanism is called the grain-boundary-limited conduction $[10,34]$. The grain boundary will build a grain boundary potential energy barrier $\left(\Phi_{B}\right)$ which is inversely proportional to the relative dielectric constant of the dielectric material. The potential energy barrier can be written as

$$
\Phi_{B}=q \phi_{B}=\frac{q^{2} n_{b}^{2}}{2 \varepsilon N},
$$

where $n_{b}$ is the grain boundary trap density, $\varepsilon$ is the relative dielectric constant of the dielectric material, and $N$ is the dopant concentration. From (27), it can be seen that the dielectric constant can significantly affect the potential energy barrier at the grain boundaries.

Figure 29(a) shows the charge distribution across an electron-trapped grain boundary and the existence of depletion regions next to the grain boundary. The potential energy barrier at the grain boundary is shown in Figure 29(b) due to the charge distribution close to the grain boundary. Figure 30 indicates an energy band diagram of the grain-boundarylimited conduction in a metal-insulator-metal MIM diode.

\section{Summary}

The conduction mechanisms in dielectric films are discussed in detail in this review. There are two types of conduction 


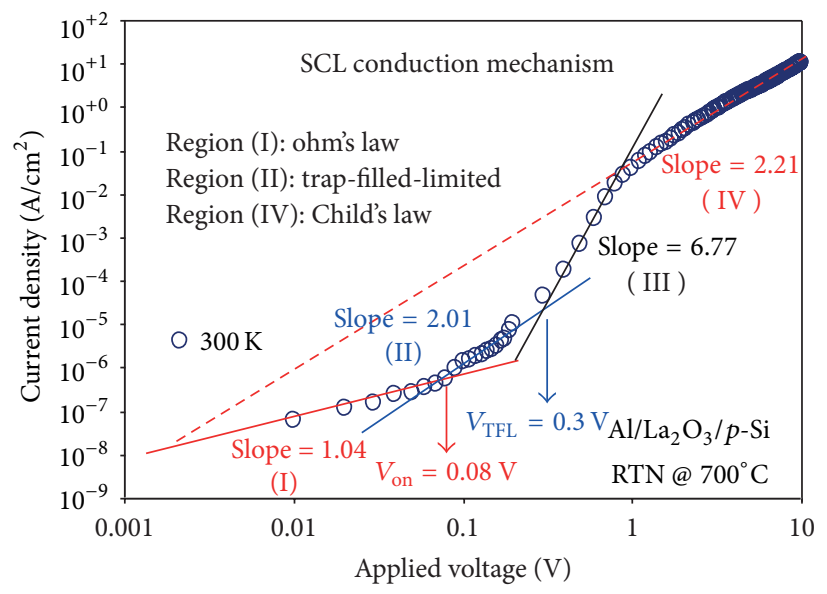

FIGURE 27: Logarithm of the current density plotted as a function of the logarithm of the applied voltage under negative bias at room temperature.

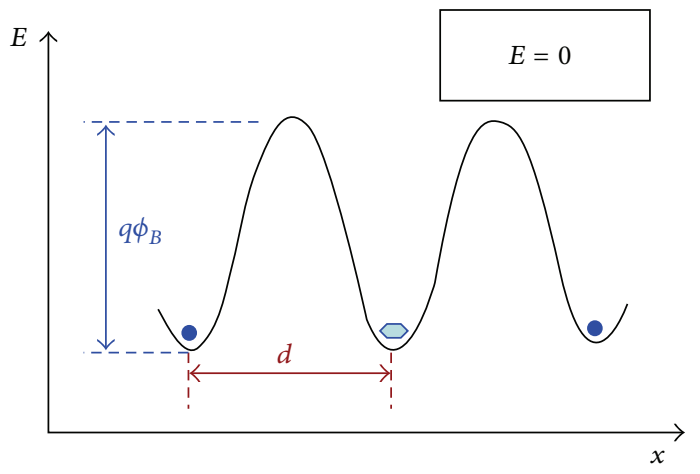

(a)

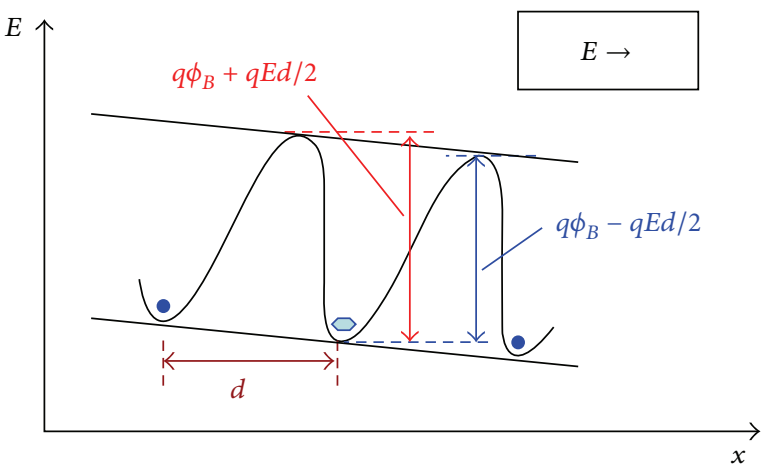

(b)

FIGURE 28: Energy band diagram of ionic conduction (a) without the applied electric field and (b) with the applied electric field. $E$ is the applied electric field, $d$ is the spacing between ionic sites, and $q \phi_{B}$ is the potential barrier height.

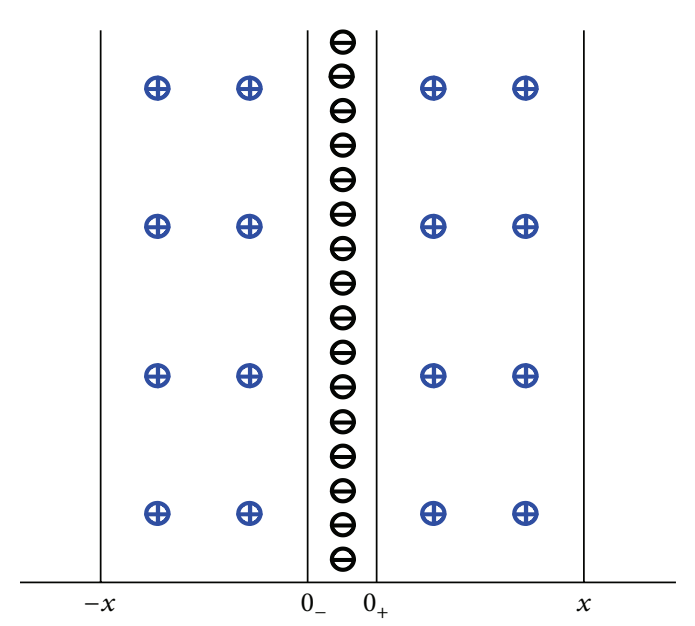

(a)

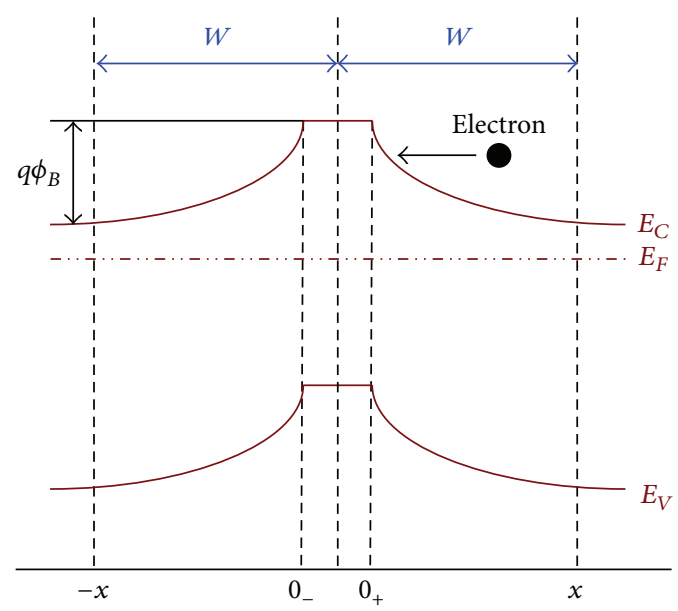

(b)

FIGURE 29: (a) The charge distribution of an electron trapping grain boundary and (b) the resulting potential energy barrier at the grain boundary. $q \phi_{B}$ is the potential barrier and $W$ is the depletion width. 


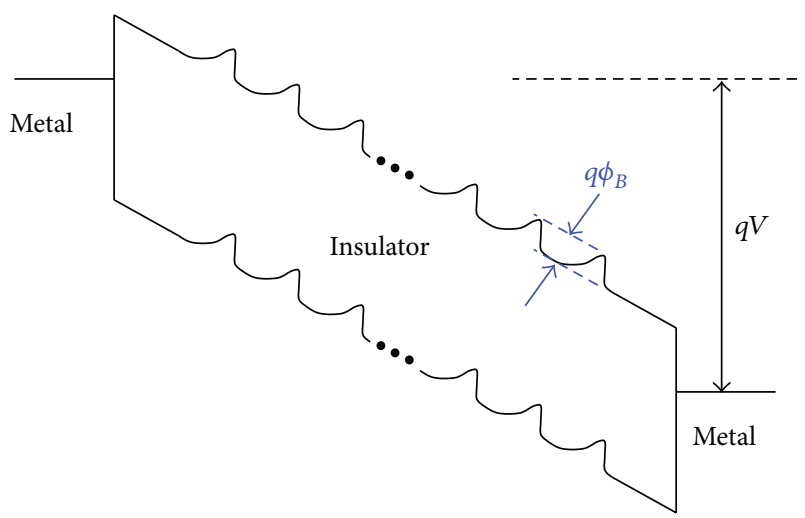

FIGURE 30: Schematic energy band diagram for grain-boundarylimited conduction in metal-insulator-metal structure.

mechanisms in dielectric films, that is, electrode-limited conduction mechanism and bulk-limited conduction mechanism. The most important parameter in the electrode-limited conduction mechanisms is the barrier height at the electrodedielectric interface; meanwhile, the most important parameter in the bulk-limited conduction mechanisms is the trap energy level in dielectric films. During the analysis of conduction mechanisms, the dielectric constant is a key factor. For the case of thermionic emission process, the dielectric constant should be equal to the optical dielectric constant if the electron transit time is shorter than the dielectric relaxation time. Based on the electrode-limited conduction mechanisms, the physical properties of the barrier height at the electrode-dielectric interface and the effective mass of the conduction carriers in dielectric films can be obtained. Similarly, based on the bulk-limited conduction mechanisms, the physical properties of the trap level, the trap spacing, the trap density, the carrier drift mobility, the dielectric relaxation time, and the density of states of the conduction band in dielectric films can be determined. In general, the conduction mechanism in dielectric films may be influenced by the following factors: temperature, electric field, stress condition, device structure (MIS or MIM), electrode material, film species $\left(\mathrm{SiO}_{2}\right.$, high-dielectric-constant material, or ferroelectric material), film thickness, deposition method, and so on. Because all the mentioned factors can affect the dielectricelectrode interface and/or the dielectric bulk property, all the factors are important in the studies of dielectric conduction mechanisms.

\section{Conflict of Interests}

The author declares that there is no conflict of interests regarding the publication of this paper.

\section{Acknowledgment}

The author would like to thank the National Science Council of the Republic of China, Taiwan, for supporting this work under Contract no. NSC 102-2221-E-130-015-MY2.

\section{References}

[1] D. R. Lamb, Electrical Conduction Mechanisms in Thin Insulating Films, Methuen, London, UK, 1967.

[2] M. A. Lambert and P. Mark, Current Injection in Solids, Academic Press, New York, NY, USA, 1970.

[3] J. G. Simmons, "Electronic conduction through thin insulating films," in Handbook of Thin Film Technology, L. Maissel and R. Glang, Eds., chapter 14, McGraw-Hill, New York, NY, USA, 1970.

[4] J. J. O'Dwyer, The Theory of Electrical Conduction and Breakdown in Solid Dielectrics, Clarendon Press, Oxford, UK, 1973.

[5] N. F. Mott and E. A. Davis, Electronic Processes in Non-Crystalline Materials, Oxford University Press, Oxford, UK, 1979.

[6] K. C. Kao and W. Hwang, Electrical Transport in Solids, Pergamon Press, New York, NY, USA, 1981.

[7] P. Hesto, "The nature of electronic conduction in thin insulating films," in Instabilities in Silicon Devices: Silicon Passivation and Related Instabilities, G. Barbottin and A. Vapaille, Eds., Elsevier Science, North Holland, Amsterdam, The Netherlands, 1st edition, 1986.

[8] C. Hamann, H. Burghardt, and T. Frauenheim, Electrical Conduction Mechanisms in Solids, VEB Deutscher Verlag der Wissenschaften, Berlin, Germany, 1988.

[9] K. C. Kao, Dielectric Phenomena in Solids, Academic Press, New York, NY, USA, 2004.

[10] J. Y. M. Lee, F. C. Chiu, and P. C. Juan, "The application of highdielectric-constant and ferroelectric thin filmsin integrated circuit technology," in Handbook of Nanoceramics and Their Based Nanodevices, T. Y. Tseng and H. S. Nalwa, Eds., vol. 4, American Scientific Publishers, Stevenson Ranch, Calif, USA, 2009.

[11] F. C. Chiu and C. M. Lai, "Optical and electrical characterizations of cerium oxide thin films," Journal of Physics D, vol. 43, no. 7, Article ID 075104, 5 pages, 2010.

[12] W.-C. Lee and C. Hu, "Modeling CMOS tunneling currents through ultrathin gate oxide due to conduction-and valenceband electron and hole tunneling," IEEE Transactions on Electron Devices, vol. 48, no. 7, pp. 1366-1373, 2001.

[13] F.-C. Chiu, Z.-H. Lin, C.-W. Chang et al., "Electron conduction mechanism and band diagram of sputter-deposited $\mathrm{Al} / \mathrm{ZrO} \mathrm{r}_{2} / \mathrm{Si}$ structure," Journal of Applied Physics, vol. 97, no. 3, Article ID 034506, 4 pages, 2005.

[14] J. G. Simmons, "Richardson-schottky effect in solids," Physical Review Letters, vol. 15, no. 25, pp. 967-968, 1965.

[15] S. Zafar, R. E. Jones, B. Jiang, B. White, V. Kaushik, and S. Gillespie, "The electronic conduction mechanism in barium strontium titanate thin films," Applied Physics Letters, vol. 73, no. 24, pp. 3533-3535, 1998.

[16] F.-C. Chiu, "Interface characterization and carrier transportation in metal/ $\mathrm{HfO}_{2} /$ silicon structure," Journal of Applied Physics, vol. 100, no. 11, Article ID 114102, 5 pages, 2006.

[17] D. K. Schroder, Semiconductor Material and Device Characterizationedition, John Wiley \& Sons, New York, NY, USA, 2nd edition, 1998.

[18] K. Khairurrijal, W. Mizubayashi, S. Miyazaki, and M. Hirose, "Analytic model of direct tunnel current through ultrathin gate oxides," Journal of Applied Physics, vol. 87, no. 6, pp. 3000-3005, 2000.

[19] M. Städele, F. Sacconi, A. Di Carlo, and P. Lugli, "Enhancement of the effective tunnel mass in ultrathin silicon dioxide layers," Journal of Applied Physics, vol. 93, no. 5, pp. 2681-2690, 2003. 
[20] B. Brar, G. D. Wilk, and A. C. Seabaugh, “Thickness and effective electron mass measurements for thin silicon dioxide films using tunneling current oscillations," Applied Physics Letters, vol. 67, no. 7, pp. 1031-1033, 1995.

[21] M. Städele, B. R. Tuttle, and K. Hess, "Tunneling through ultrathin $\mathrm{SiO}_{2}$ gate oxides from microscopic models," Journal of Applied Physics, vol. 89, no. 1, pp. 348-363, 2001.

[22] Y.-C. Yeo, T.-J. King, and C. Hu, "Direct tunneling leakage current and scalability of alternative gate dielectrics," Applied Physics Letters, vol. 81, no. 11, pp. 2091-2093, 2002.

[23] F.-C. Chiu, C.-Y. Lee, and T.-M. Pan, "Current conduction mechanisms in $\operatorname{Pr}_{2} \mathrm{O}_{3}$ /oxynitride laminated gate dielectrics," Journal of Applied Physics, vol. 105, no. 7, Article ID 074103, 4 pages, 2009.

[24] R. L. Angle and H. E. Talley, "Electrical and charge storage characteristics of the tantalum oxide-silicon dioxide device," IEEE Transactions on Electron Devices, vol. 25, no. 11, pp. 1277-1283, 1978.

[25] F. C. Chiu, W. C. Shih, and J. J. Feng, "Conduction mechanism of resistive switching films in $\mathrm{MgO}$ memory devices," Journal of Applied Physics, vol. 111, no. 9, Article ID 094104, 5 pages, 2012.

[26] H. Akinaga and H. Shima, "Resistive random access memory (ReRAM) based on metal oxides," Proceedings of the IEEE, vol. 98, no. 12, pp. 2237-2251, 2010.

[27] A. Sawa, "Resistive switching in transition metal oxides," Materials Today, vol. 11, no. 6, pp. 28-36, 2008.

[28] R. Waser, R. Dittmann, C. Staikov, and K. Szot, "Redoxbased resistive switching memories nanoionic mechanisms, prospects, and challenges," Advanced Materials, vol. 21, no. 2526, pp. 2632-2663, 2009.

[29] F.-C. Chiu, P.-W. Li, and W.-Y. Chang, "Reliability characteristics and conduction mechanisms in resistive switching memory devices using ZnO thin films," Nanoscale Research Letters, vol. 7, Article ID 178, 9 pages, 2012.

[30] S. M. Sze and K. K. Ng, Physics of Semiconductor Devices, John Wiley \& Sons, Hoboken, NJ, USA, 3rd edition, 2006.

[31] M. Nakano, A. Tsukazaki, R. Y. Gunji et al., "Schottky contact on a $\mathrm{ZnO}$ (0001) single crystal with conducting polymer," Applied Physics Letters, vol. 91, no. 14, Article ID 142113, 3 pages, 2007.

[32] F.-C. Chiu, H.-W. Chou, and J. Y.-M. Lee, "Electrical conduction mechanisms of metal $\mathrm{La}_{2} \mathrm{O}_{3}$ Si structure," Journal of Applied Physics, vol. 97, no. 10, Article ID 103503, 5 pages, 2005.

[33] F.-C. Chiu, "Electrical characterization and current transportation in metal $\mathrm{Dy}_{2} \mathrm{O}_{3}$ Si structure," Journal of Applied Physics, vol. 102, no. 4, Article ID 044116, 5 pages, 2007.

[34] T. K. Kundu and J. Y.-M. Lee, “Thickness-dependent electrical properties of $\mathrm{Pb}(\mathrm{Zr}, \mathrm{Ti}) \mathrm{O}_{3}$ thin film capacitors for memory device applications," Journal of the Electrochemical Society, vol. 147, no. 1, pp. 326-329, 2000. 

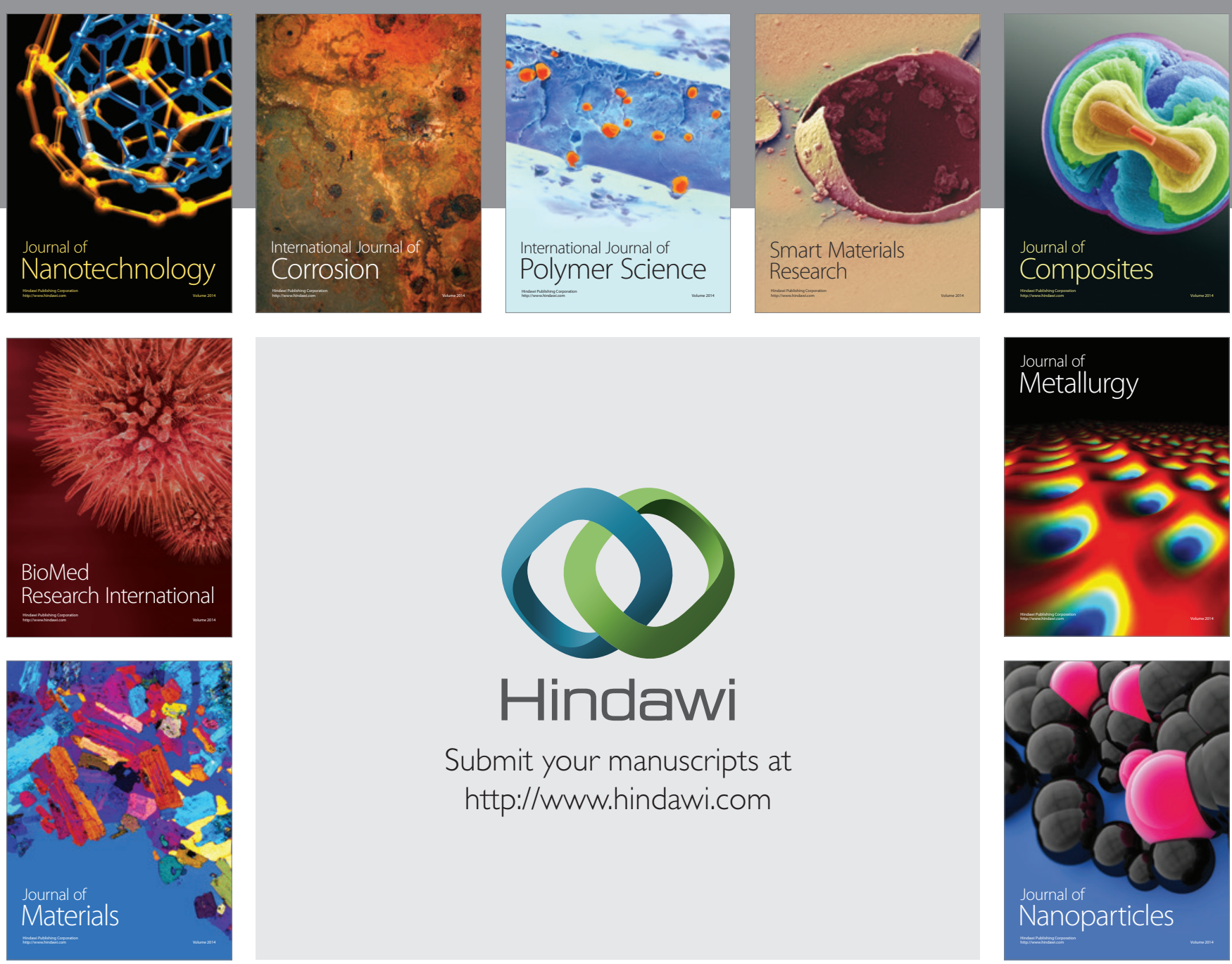

Submit your manuscripts at http://www.hindawi.com
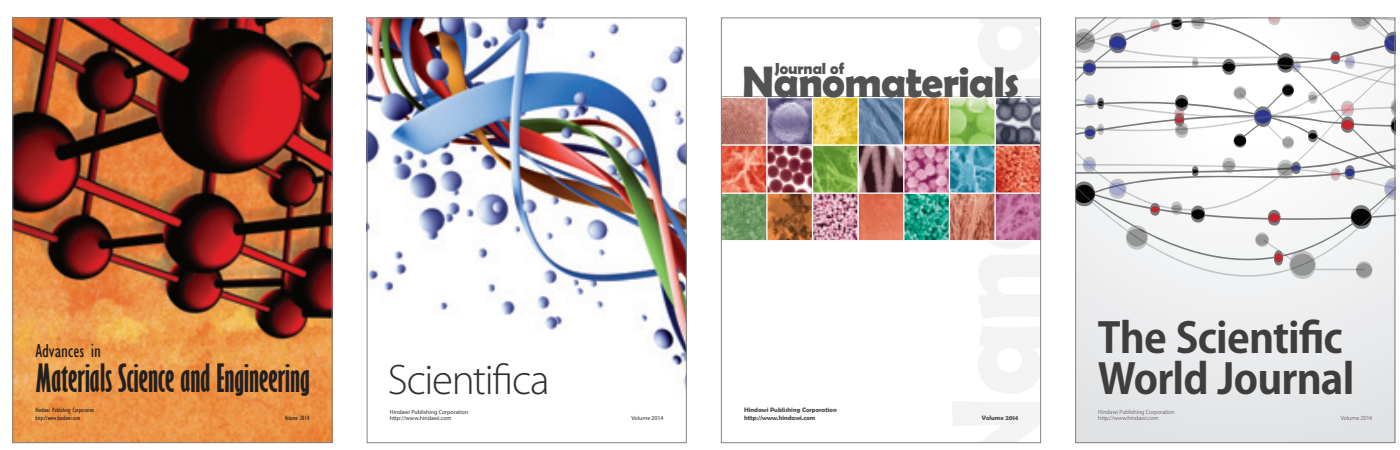

\section{The Scientific World Journal}
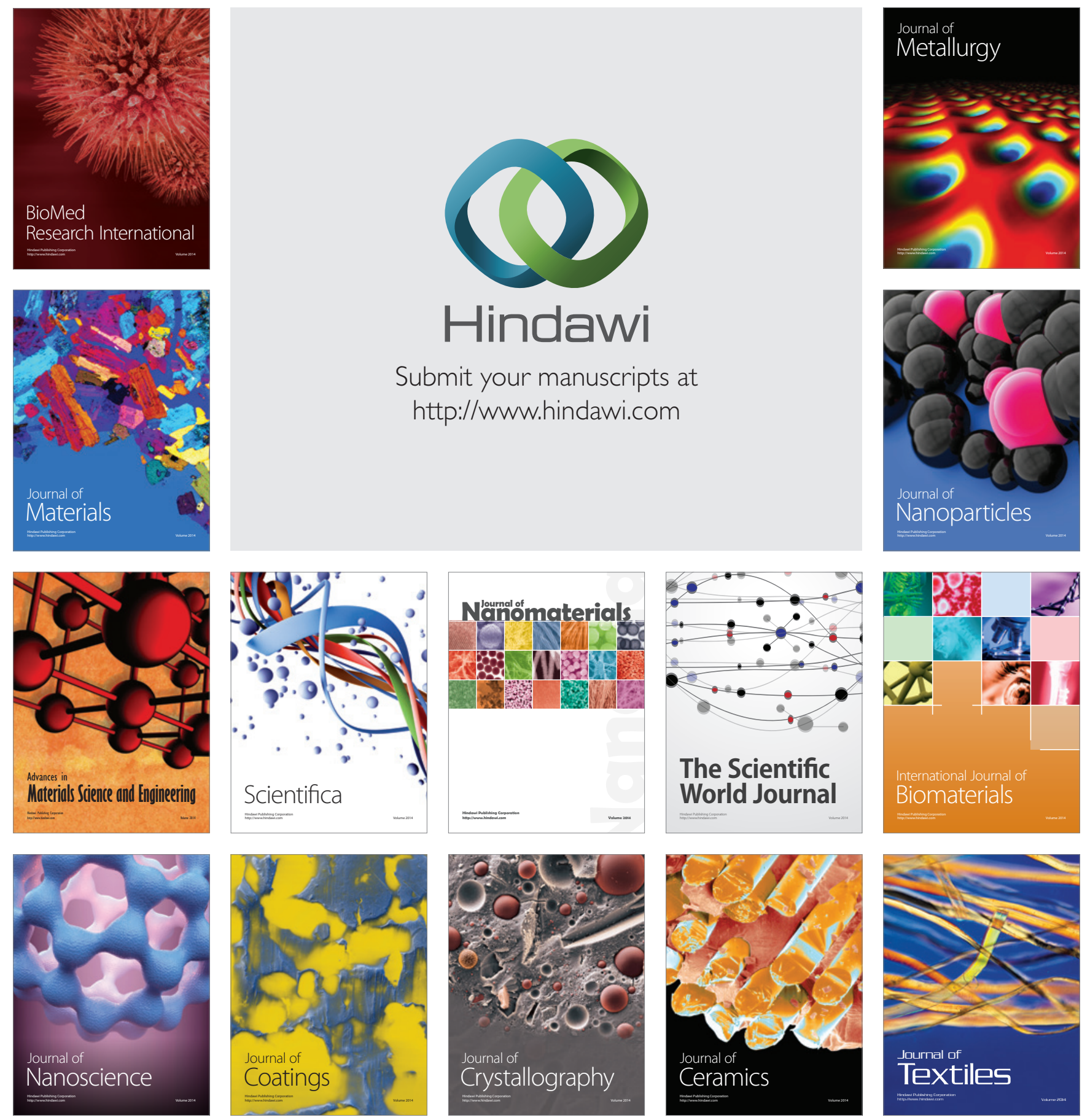